\title{
Moduli Spaces of Affine Homogeneous Spaces
}

\author{
Gregor Weingart*
}

July 20th, 2017

\begin{abstract}
Apart from global topological problems an affine homogeneous space is locally described by its curvature, its torsion and a slightly less tangible object called its connection in a given base point. Using this description of the local geometry of an affine homogeneous space we construct an algebraic variety $\mathfrak{M}(\mathfrak{g l} V)$, which serves as a coarse moduli space for the local isometry classes of affine homogeneous spaces of dimension $\operatorname{dim} V$. Moreover we associate a Sym $V^{*}$-comodule to a point in $\mathfrak{M}(\mathfrak{g l} V)$ and use its Spencer cohomology in order to describes the infinitesimal deformations of this point in the true moduli space $\mathfrak{M}_{\infty}(\mathfrak{g l} V)$.
\end{abstract}

\section{MSC2010: 53C30; 22F30}

\section{Introduction}

Homogeneous spaces comprise a class of smooth manifolds of particular interest in differential geometry, because many geometric calculations reduce essencially to linear algebra in the presence of a transitively acting Lie group. In particular all of the geometry of a homogeneous space except for its global topology can be represented in terms of converging formal power series on a formal neighborhood of the base point. This representation of the local geometry of a homogeneous space by convergent power series will be used implicitly in this article to construct moduli spaces of isometry classes of and a corresponding deformation theory for affine homogeneous spaces with or without additional geometric structures.

A particular advantage of our approach in comparison to the moduli spaces of locally homogeneous spaces constructed in [Ts] is that the covariant derivatives of the curvature and/or the torsion in the construction of the moduli spaces are replaced by the Christoffel symbols of the connection in the base point. The locally affine homogeneous spaces considered in $[\mathrm{S}][\mathrm{K} 1][\mathrm{O}][\mathrm{Tr}][\mathrm{Ts}]$ for example can be thought of as affine homogeneous spaces with variable Christoffel symbols or equivalently as smooth maps to the moduli spaces constructed below, the derivatives of these maps can thus be studied using the deformation theory of

\footnotetext{
*Instituto de Matemáticas (Cuernavaca), Universidad Nacional Autónoma de México, Avenida Universidad s/n, Colonia Lomas de Chamilpa, 62210 Cuernavaca, Morelos, MEXICO; gw@matcuer.unam.mx
} 
affine homogeneous spaces arising naturally in our approach. Put differently the examples of locally, but not globally affine homogeneous spaces owe their existence to the non-vanishing of certain Spencer cohomology spaces, which replace the Chevalley-Eilenberg cohomology spaces in the deformation theory of Lie algebras.

Representing the local geometry of an affine homogeneous spaces in terms of converging formal power series obliterates its global topology as a manifold, hence we will essentially consider only formal affine homogeneous spaces defined as a pair $\mathfrak{g} \supset \mathfrak{h}$ of Lie algebras endowed with an $\mathfrak{h}$-equivariant linear map $A: \mathfrak{g} \longrightarrow$ End $\mathfrak{g} / \mathfrak{h}$ extending the isotropy representation $\star: \mathfrak{h} \longrightarrow$ End $\mathfrak{g} / \mathfrak{h}$. This algebraic simplicification is bought at a prize however: The integration of a formal affine homogeneous space into a real manifold will fail in general to produce an affine homogeneous space, because the Lie algebra $\mathfrak{h}$ may not integrate to a closed subgroup of the simply connected Lie group $G$ corresponding to the Lie algebra $\mathfrak{g}$. Nevertheless every formal affine homogeneous space corresponds to a homogeneous transitive Lie algebroid over a homogeneous space endowed with a left invariant connection as discussed briefly in the comments after Definition 3.1.

In order to construct the moduli spaces of formal affine homogeneous spaces we fix a model vector space $V$ of the appropiate dimension and augment a given formal affine homogeneous space $\mathfrak{g} \supset \mathfrak{h}$ endowed with $A: \mathfrak{g} \longrightarrow$ End $\mathfrak{g} / \mathfrak{h}$ by an isomorphism or frame $F: V \longrightarrow \mathfrak{g} / \mathfrak{h}$ and a not necessarily $\mathfrak{h}$-equivariant split $\mathfrak{g} / \mathfrak{h} \longrightarrow \mathfrak{g}$ of the canonical projection. With these two additional pieces of data in place we may associate a connection-curvature-torsion triple

$$
(A, R, T) \in V^{*} \otimes \text { End } V \times \Lambda^{2} V^{*} \otimes \text { End } V \times \Lambda^{2} V^{*} \otimes V
$$

to an augmented formal affine homogeneous space, which encodes the geometry of $\mathfrak{g} / \mathfrak{h}$ completely. Our main result characterizes the subset $\mathfrak{M}(\mathfrak{g l} V)$ of connection-curvature-torsion triples arising from augmented formal affine homogeneous spaces by means of a formally infinite, but actually finite system of explicit homogeneous algebraic equations:

\section{Theorem 4.8 (Algebraic Variety of Affine Homogeneous Spaces)}

$A$ connection-curvature-torsion triple $(A, R, T)$ on a vector space $V$ represents a formal affine homogeneous space $\mathfrak{g} \supset \mathfrak{h}$ augmented by a frame isomorphism $F: V \longrightarrow \mathfrak{g} / \mathfrak{h}$ and a split $\mathfrak{g} / \mathfrak{h} \longrightarrow \mathfrak{g}$ of the canonical projection, if and only if $(A, R, T)$ satisfies the formal first and second Bianchi identities $d^{(A, T)} T=R \wedge$ id and $d^{(A, T)} R=0$ of degrees 2 , 3 respectively and for all $r \geq 0$ the following homogeneous equations of degrees $r+3$ and $r+4$

$$
\begin{aligned}
& (Q(A, T)-R) \circledast(\underbrace{A \circledast(A \circledast(\ldots(A}_{r \text { times }} \circledast T) \ldots)))=0 \\
& (Q(A, T)-R) \circledast(\underbrace{A \circledast(A \circledast(\ldots(A}_{r \text { times }} \circledast R) \ldots)))=0
\end{aligned}
$$

where $Q(A, T)_{x, y}:=\left[A_{x}, A_{y}\right]-A_{A_{x} y-A_{y} x-T(x, y)}$ is the approximate curvature of $(A, R, T)$. This result generalizes the well-known description of local isometry classes of symmetric spaces in terms of their curvature $R[\mathrm{H}]$ and the classification of manifolds with parallel 
curvature and torsion by Ambrose and Singer [AS]. Eventually we will introduce the equivalence relation of infinite order contact $\sim_{\infty}$ on connection-curvature-torsion triples, which describes precisely the effect of changing the frame $F$ and/or the split $\mathfrak{g} / \mathfrak{h} \longrightarrow \mathfrak{g}$. The true moduli space of isometry classes of formal affine homogeneous spaces is thus the quotient:

$$
\mathfrak{M}_{\infty}(\mathfrak{g l} V):=\mathfrak{M}(\mathfrak{g l} V) / \sim_{\infty}
$$

A subtle invariant of a connection-curvature-torsion triple $(A, R, T) \in \mathfrak{M}(\mathfrak{g l} V)$ plays a prominent role in the proof of Theorem 4.8, the stabilizer filtration of End $V$ by subalgebras

$$
\text { End } V=\ldots=\mathfrak{h}_{-2}=\mathfrak{h}_{-1} \supsetneq \mathfrak{h}_{0} \supsetneq \ldots \supsetneq \mathfrak{h}_{s-1} \supsetneq \mathfrak{h}_{s}=\mathfrak{h}_{s+1}=\ldots=\mathfrak{h}_{\infty}
$$

introduced by Singer [S], in particular the minimal $s \geq-1$ with equality $\mathfrak{h}_{s}=\mathfrak{h}_{s+1}$ is nowadays called the Singer invariant of $(A, R, T)$. Our recursive Definition 4.4 of the stabilizer filtration differs significantly from Singer's definition akin to Lemma 4.5 and is certainly easier to use in actual calculations. The graded vector space of sucessive filtration quotients in the stabilizer filtration associated to a connection-curvature-torsion triple

$$
\mathfrak{h}^{\bullet}:=\bigoplus_{r \in \mathbb{Z}}\left(\mathfrak{h}_{r-1} / \mathfrak{h}_{r}\right)=\left(\text { End } V / \mathfrak{h}_{0}\right) \oplus \ldots \oplus\left(\mathfrak{h}_{s-1} / \mathfrak{h}_{\infty}\right)
$$

is naturally a Sym $V^{*}$-comodule and thus allows us to associate a cohomology theory to every point $(A, R, T)$ in the moduli space $\mathfrak{M}(\mathfrak{g l} V)$, namely the Spencer cohomology $H^{\bullet, \circ}(\mathfrak{h})$ of the comodule $\mathfrak{h}^{\bullet}$. The second main result of this article links the special Spencer cohomology spaces $H^{\bullet}, 1(\mathfrak{h})$ to a geometric filtration on the formal tangent space to the true moduli space $\mathfrak{M}_{\infty}(\mathfrak{g l} V)$ in the point represented by the connection-curvature-torsion triple $(A, R, T)$ :

$$
H^{\bullet}, 1(\mathfrak{h})=T_{[A, R, T]} \mathfrak{M}_{\infty}^{\bullet}(A, R, T) / T_{[A, R, T]} \mathfrak{M}_{\infty}^{\bullet-1}(A, R, T)
$$

Philosophically this equality reflects the simple fact that a vector field representing an $r$-jet solution to the affine Killing equation, which can not be lifted to an $r+1$-jet solution, can not be an affine Killing field, hence its flow will change the underlying geometry, but gently enough to stay in contact with the original geometry up to order $r-2$. In a rather precise sense equation (1) is a quantitative version of Singer's Theorem [S], which characterizes the globally among the locally Riemannian homogeneous spaces, because the Spencer cohomology spaces are trivial by construction for degrees $\bullet>s$ larger than the Singer invariant so that no further deformations are possible. A paradoxical aspect of the preceeding construction is that Spencer cohomology is usually introduced to describe the set of $r+1$-jet solutions as an affine space bundle over the set of $r$-jet solutions, however it works just as well for the affine Killing equation, where the $r$-jet solutions are an affine space over the $r+1$-jet solutions.

In Section 2 of this article we will recall many well-known facts about homogeneous spaces, in particular we will discuss a very convenient calculus for left invariant connections on homogeneous vector bundles. Section 3 describes two equivalent ways to encode the local geometry of a formal affine homogeneous space in an algebra endowed with a skew bracket, one of these 
algebras has been studied in $[\mathrm{NT}]$. In Section 4 we introduce connection-curvature-torsion triples and derive the algebraic equations defining of the coarse moduli space $\mathfrak{M}(\mathfrak{g l} V)$ as an algebraic variety. Additional parallel geometric structures like Riemannian metrics or almost complex structures are added to the picture in Section 5, whereas the final Section 6 constructs the Spencer cohomology of a formal affine homogeneous space and calculates this cohomology for the family of examples of Riemannian homogeneous spaces with large Singer invariant constructed by C. Meusers $[\mathrm{M}]$, which turns out to be a maximal family in the sense that it is closed under all its possible deformations.

The framework of this article was developped in intensive collaboration with C. Meusers, the recursive definition of the stabilizer filtration for example arose directly from these discussion and was subsequently published in $[\mathrm{M}]$. Besides C. Meusers the author would like to thank W. Ballmann for his support and encouragement in writing up this article.

\section{Left Invariant Connections}

Certainly the most important single concept in studying the geometry of a homogeneous space $G / H$ is the notion of a homogeneous vector bundle over $G / H$, a vector bundle endowed with a left $G$-action on its total space, which covers the canonical left action of $G$ on $G / H$ and is linear in every fiber. The additional $G$-action on the total space makes the vector space of sections of a homogeneous vector bundle a representation of the group $G$ generalizing the left regular representation of $G$ on $C^{\infty}(G)$. A differential operator between sections of homogeneous vector bundles commuting with the respective representations of $G$ is called for this reason a left invariant differential operator. In this section we will focus on the algebraic properties of a specific subclass of left invariant differential operators, left invariant connections. The detailed algebraic formalism introduced in this section to describe left invariant connections will turn out to be quite useful for our subsequent calculations.

Recall that a homogeneous vector bundle on a homogeneous space $G / H$ is a vector bundle on which $G$ acts from the left by vector bundle morphisms covering the left action of $G$ on $G / H$. General nonsense implies that the category of homogeneous vector bundles over $G / H$ under $G$-equivariant vector bundle homomorphisms is equivalent to the category of representations of $H$ under $H$-equivariant linear maps. One possible choice for the $H$-representation in this correspondence is simply the fiber $\Sigma:=\Sigma_{e H}(G / H)$ of the homogeneous vector bundle $\Sigma(G / H)$ over the base point $e H \in G / H$, which is a representation of $H$ by the very definition of a homogeneous vector bundle. For the tangent and cotangent bundles however these fibers are usually replaced by the representations $\mathfrak{g} / \mathfrak{h}$ and $(\mathfrak{g} / \mathfrak{h})^{*}$, which are isomorphic to the fibers $T_{e H}(G / H)$ and $T_{e H}^{*}(G / H)$ respectively via the $H$-equivariant isomorphism:

$$
\mathfrak{g} / \mathfrak{h} \stackrel{\cong}{\longrightarrow} T_{e H}(G / H), \quad X+\left.\mathfrak{h} \longmapsto \frac{d}{d t}\right|_{0} e^{t X} H
$$

Definition 2.1 (Homogeneous Vector Bundles)

A homogeneous vector bundle on a homogeneous space $G / H$ is a vector bundle $\Sigma(G / H)$ on 
$G / H$ endowed with a left $G$-action on its total space, which covers the canonical left action $\star: G \times G / H \longrightarrow G / H,(\gamma, g H) \longmapsto \gamma g H$, of $G$ on $G / H$ and is linear in every fiber:

$$
\star: \quad G \times \Sigma(G / H) \longrightarrow \Sigma(G / H), \quad(\gamma, s) \longmapsto \gamma \star s
$$

The vector space of sections of a homogeneous vector bundle becomes a representation of $G$

$$
L: \quad G \times \Gamma \Sigma(G / H) \longrightarrow \Gamma \Sigma(G / H), \quad(\gamma, s) \longmapsto L_{\gamma} s
$$

by means of $\left(L_{\gamma} s\right)(g H):=\gamma \star s\left(\gamma^{-1} g H\right)$ for all $\gamma \in G$ and all sections $s \in \Gamma \Sigma(G / H)$.

In the same vein a homogeneous fiber bundle over a homogeneous space $G / H$ is a fiber bundle over $G / H$ endowed with a left action of $G$ on its total space, which covers the canonical left action on $G / H$. In particular the group $G$ itself can be considered as a homogeneous principal $H$-bundle over $G / H$, where the commuting left and right multiplications in

$$
G \times G \times H \longrightarrow G, \quad(\gamma, g, h) \longmapsto \gamma g h
$$

define the left $G$-action on the total space and the principal $H$-bundle structure respectively. In turn a left invariant principal connection is a differential form $\omega \in \Gamma\left(T^{*} G \otimes \mathfrak{h}\right)$ on $G$ with values in $\mathfrak{h}$ invariant under the left $G$-action, such that the axiom for principal $H$-connections

$$
\omega_{g_{0} h_{0}}\left(\left.\frac{d}{d t}\right|_{0} g_{t} h_{t}\right)=\operatorname{Ad}_{h_{0}^{-1}} \omega_{g_{0}}\left(\left.\frac{d}{d t}\right|_{0} g_{t}\right)+\left.\frac{d}{d t}\right|_{0} h_{0}^{-1} h_{t}
$$

is satisfied for all curves $t \longmapsto g_{t}$ in $G$ and $t \longmapsto h_{t}$ in $H$. The Maurer-Cartan form $\theta \in \Gamma\left(T^{*} G \otimes \mathfrak{g}\right)$ provides a left invariant trivialization of $T G$, hence every left invariant connection is necessarily of the form $\omega \circ \theta$ for a linear map $\omega: \mathfrak{g} \longrightarrow \mathfrak{h}$. However the principal connection axiom (2) requires $\omega$ to be an $H$-equivariant section of the inclusion:

\section{Definition 2.2 (Left Invariant Principal Connections)}

A left invariant principal connection on $G$ considered as a homogeneous principal $H$-bundle over $G / H$ is an $H$-equivariant section $\omega: \mathfrak{g} \longrightarrow \mathfrak{h}$ of the short exact sequence:

$$
0 \longrightarrow \mathfrak{h} \longrightarrow \mathfrak{g} \longrightarrow \mathfrak{g} / \mathfrak{h} \longrightarrow 0
$$

In consequence of this definition the set of left invariant principal connections on a given homogeneous space $G / H$ is either the empty set or an affine space modelled on $\operatorname{Hom}_{H}(\mathfrak{g} / \mathfrak{h}, \mathfrak{h})$. In fact the set of all left invariant principal connections on $G / H$ is precisely the preimage of $\operatorname{id}_{\mathfrak{h}} \in \operatorname{Hom}_{H}(\mathfrak{h}, \mathfrak{h})$ under the map $\operatorname{Hom}_{H}(\mathfrak{g}, \mathfrak{h}) \longrightarrow \operatorname{Hom}_{H}(\mathfrak{h}, \mathfrak{h})$ in the long exact sequence

$$
0 \longrightarrow \operatorname{Hom}_{H}(\mathfrak{g} / \mathfrak{h}, \mathfrak{h}) \longrightarrow \operatorname{Hom}_{H}(\mathfrak{g}, \mathfrak{h}) \longrightarrow \operatorname{Hom}_{H}(\mathfrak{h}, \mathfrak{h}) \stackrel{\delta}{\longrightarrow} \operatorname{Ext}_{H}^{1}(\mathfrak{g} / \mathfrak{h}, \mathfrak{h}) \longrightarrow
$$

associated to the short exact sequence of $H$-representations:

$$
0 \longrightarrow \mathfrak{h} \longrightarrow \mathfrak{g} \longrightarrow \mathfrak{g} / \mathfrak{h} \longrightarrow 0
$$


The extension class $\delta\left(\operatorname{id}_{\mathfrak{h}}\right) \in \operatorname{Ext}_{H}^{1}(\mathfrak{g} / \mathfrak{h}, \mathfrak{h})$ is thus the unique obstruction against the existence of a left invariant principal connection on a homogeneous space $G / H$. A homogenous space $G / H$ with vanishing $\delta\left(\mathrm{id}_{\mathfrak{h}}\right)=0$ is properly called a reductive homogeneous space, in the literature however this notion implicitly includes the choice of some left invariant principal connection $\omega: \mathfrak{g} \longrightarrow \mathfrak{h}$ or other. Concealing this arbitrary choice in the inconspicuous adjective reductive it is somewhat duplicitous though to call the induced connections on homogeneous vector bundles canonical connections.

With the cotangent bundle $T^{*}(G / H)$ of a homogeneous space $G / H$ being homogeneous the group $G$ acts not only on the vector space of sections $\Gamma(\Sigma(G / H))$ of a homogeneous vector bundle $\Sigma(G / H)$, but also on $\Gamma\left(T^{*}(G / H) \otimes \Sigma(G / H)\right)$. In turn a linear connection

$$
\nabla: \quad \Gamma(\Sigma(G / H)) \longrightarrow \Gamma\left(T^{*}(G / H) \otimes \Sigma(G / H)\right)
$$

on $\Sigma(G / H)$ is called a left invariant connection on $\Sigma(G / H)$ provided $\nabla$ is $G$-equivariant in the sense $L_{\gamma}(\nabla s)=\nabla\left(L_{\gamma} s\right)$ for all sections $s \in \Gamma(\Sigma(G / H))$ and every $\gamma \in G$. For the purpose of this article we want to replace this definition by a more algebraic formulation:

\section{Definition 2.3 (Left Invariant Connections)}

A left invariant connection on a homogeneous vector bundle $\Sigma(G / H)$ is an $H$-equivariant extension $A: \mathfrak{g} \longrightarrow$ End $\Sigma$ of the infinitesimal representation $\star: \mathfrak{h} \longrightarrow$ End $\Sigma$ of $\mathfrak{h}$ on $\Sigma$.

Similar to the classification of left invariant principal connections the set of left invariant connections $A: \mathfrak{g} \longrightarrow$ End $\Sigma$ on $\Sigma(G / H)$ is the preimage of the infinitesimal representation $\star: \mathfrak{h} \longrightarrow$ End $\Sigma$ under $\operatorname{Hom}_{H}(\mathfrak{g}$, End $\Sigma) \longrightarrow \operatorname{Hom}_{H}(\mathfrak{h}$, End $\Sigma)$ in the long exact sequence:

$$
\ldots \longrightarrow \operatorname{Hom}_{H}(\mathfrak{g}, \text { End } \Sigma) \longrightarrow \operatorname{Hom}_{H}(\mathfrak{h}, \text { End } \Sigma) \stackrel{\delta}{\longrightarrow} \operatorname{Ext}_{H}^{1}(\mathfrak{g} / \mathfrak{h}, \text { End } \Sigma) \longrightarrow \ldots
$$

In consequence the existence of left invariant connections $A: \mathfrak{g} \longrightarrow$ End $\Sigma$ on $\Sigma(G / H)$ is obstructed by the extension class $\delta(\star) \in \operatorname{Ext}_{H}^{1}(\mathfrak{g} / \mathfrak{h}$, End $\Sigma)$, its vanishing provides us with an affine space of left invariant connections on $\Sigma(G / H)$ modelled on $\operatorname{Hom}_{H}(\mathfrak{g} / \mathfrak{h}$, End $\Sigma)$. Evidently every left invariant principal connection $\omega: \mathfrak{g} \longrightarrow \mathfrak{h}$ induces a left invariant connection $A^{\omega}: \mathfrak{g} \longrightarrow$ End $\Sigma$ on every homogeneous vector bundle $\Sigma(G / H)$ by means of $A^{\omega}(X):=\omega(X) \star$. Due to this universality every non-vanishing obstruction $\delta(\star) \neq 0$ for some $H$-representation $\Sigma$ is of course an obstruction against the existence of a left invariant principal connection as well.

For the moment we will not discuss the precise relationship between the analytic and the algebraic definition of a left invariant connection on a homogeneous vector bundle $\Sigma(G / H)$ over a homogeneous space $G / H$. Instead we want to point out a couple of very remarkable analogies, which taken together should leave no doubt that these two concepts are intimately related. The algebraic Definition 2.3 for example is easy to use with constructions of linear algebra like dual spaces, direct sums and tensor products, say the dual left invariant connection on $\Sigma^{*}$ is simply given by $-A^{*}: \mathfrak{g} \longrightarrow$ End $\Sigma^{*}, X \longmapsto-A_{X}^{*}$, whereas $A^{\Sigma} \oplus A^{\tilde{\Sigma}}$ and $A^{\Sigma} \otimes \mathrm{id}+\mathrm{id} \otimes A^{\tilde{\Sigma}}$ are left invariant connections on the direct sum $\Sigma \oplus \tilde{\Sigma}$ and tensor product $\Sigma \otimes \tilde{\Sigma}$ of two representations $\Sigma, \tilde{\Sigma}$ endowed with left invariant connections $A^{\Sigma}, A^{\tilde{\Sigma}}$. 


\section{Lemma 2.4 (Left Invariant Connections and Rigidity)}

If there exists a left invariant connection on the tangent bundle of a homogeneous space $G / H$, then the kernel $\mathfrak{n}:=\operatorname{ker} \star$ of the isotropy representation $\star: \mathfrak{h} \longrightarrow$ End $\mathfrak{g} / \mathfrak{h}, Z \longmapsto Z \star$, is an ideal not only in the isotropy algebra $\mathfrak{h}$, but already in the full Lie algebra $\mathfrak{g}$.

Proof: According to the preceeding discussion a left invariant connection on the tangent bundle can be thought of as an $H$-equivariant extension $A: \mathfrak{g} \longrightarrow$ End $\mathfrak{g} / \mathfrak{h}$ of the adjoint representation $\star$ of $\mathfrak{h}$ on $\mathfrak{g} / \mathfrak{h}$. We need to show that $[N, X] \in \mathfrak{n}$ for all $N \in \mathfrak{n}$ and $X \in \mathfrak{g}$ or equivalently $[[N, X], Y] \equiv 0 \bmod \mathfrak{h}$ for all $X, Y \in \mathfrak{g}$, because $[N, X] \in \mathfrak{h}$ by the very definition of $\mathfrak{n}$. Since the left invariant connection $A$ extends the infinitesimal representation

$$
[[N, X], Y] \equiv A_{[N, X]}(Y+\mathfrak{h}) \equiv\left[N, A_{X}(Y+\mathfrak{h})\right]-A_{X}([N, Y]+\mathfrak{h})
$$

modulo $\mathfrak{h}$, where the second congruence is the infinitesimal version of $H$-equivariance for the left invariant connection $A$ under $N \in \mathfrak{h}$. Evidently the right hand side vanishes, because $[N, Y] \in \mathfrak{h}$ and $\left[N, A_{X}(Y+\mathfrak{h})\right] \in \mathfrak{h}$ by assumption.

In general the kernel $\mathfrak{n}$ of the adjoint representation of $\mathfrak{h}$ on $\mathfrak{g} / \mathfrak{h}$ fails to be an ideal of $\mathfrak{g}$ and thus obstructs the existence of a left invariant connection on the tangent bundle and in turn a left invariant principal connection on a homogeneous space $G / H$. Homogeneous spaces $G / H$ admitting a left invariant connection on their tangent bundle are really rather special in the class of all homogeneous spaces, because $G$ will not be a subgroup of some affine group in general. Of course this simple observation does not preclude the existence of left invariant connections on other homogeneous vector bundles. The infinitesimal representation $\star: \mathfrak{g} \longrightarrow$ End $\Sigma$ of a representation $\Sigma$ of $G$ for example is always a left invariant connection on a homogeneous vector bundle over $G / H$ with base point fiber $\Sigma_{e H}(G / H)=\Sigma$.

\section{Corollary 2.5 (Kernel of the Adjoint Representation)}

If a homogeneous space $G / H$ carries a left invariant connection on its tangent bundle, then we may assume without loss of generality that the adjoint representation $\star: \mathfrak{h} \longrightarrow$ End $\mathfrak{g} / \mathfrak{h}$ is faithful. Namely under this assumption the kernel $\mathfrak{n}$ of the adjoint representation is the Lie algebra of the maximal normal subgroup $N$ of $G$ contained in $H$ so that we may present $G / H$ alternatively as $(G / N) /(H / N)$ with faithful adjoint representation $\star: \mathfrak{h} / \mathfrak{n} \longrightarrow$ End $\mathfrak{g} / \mathfrak{h}$.

Recall now that the group $G$ acts on the vector space $\Gamma(\Sigma(G / H))$ of a homogeneous vector bundle via $\left(L_{\gamma} s\right)(g H):=\gamma \star s\left(\gamma^{-1} g H\right)$, sections invariant under this representation in the sense $L_{\gamma} s=s$ for all $\gamma \in G$ are called left invariant sections. The value of such a left invariant section $s$ in the base point $e H \in G / H$ is necessarily an invariant vector in the base point fiber representation $\Sigma=\Sigma_{e H}(G / H)$, since for $h \in H$ it is necessarily true that:

$$
s(e H)=\left(L_{h} s\right)(e H)=h \star s\left(h^{-1} H\right)
$$

This argument is the central observation leading to a classification of left invariant sections: 


\section{Lemma 2.6 (Characterization of Left Invariant Sections)}

Evaluation at the base point provides an isomorphism between the vector space $\Gamma(\Sigma(G / H))^{G}$ of left invariant sections of a homogeneous vector bundle $\Sigma(G / H)$ on a homogeneous space $G / H$ and the subspace $\Sigma^{H}$ of $H$-invariant vectors in the $H$-representation $\Sigma:=\Sigma_{e H}(G / H)$ :

$$
\mathrm{ev}_{e H}: \quad[\Gamma(\Sigma(G / H))]^{G} \longrightarrow[\Sigma]^{H}, \quad s \longmapsto s(e H)
$$

The inverse isomorphism associates to $s_{e H} \in \Sigma^{H}$ the well defined section $s(g H):=g \star s_{e H}$.

With the characterization of left invariant sections at hand we can eventually make the relation between the algebraic and the analytic definition of left invariant connections somewhat more precise. Observing that the jet bundles $\operatorname{Jet}^{r} \Sigma(G / H)$ of all orders $r \geq 0$ of a homogeneous vector bundle $\Sigma(G / H)$ are naturally homogeneous under the left $G$-action

$$
\gamma \star \operatorname{jet}_{g H}^{r} s:=\operatorname{jet}_{\gamma g H}^{r}\left(L_{\gamma} s\right)=\operatorname{jet}_{\gamma g H}^{r}[\gamma \tilde{g} H \longmapsto \gamma \star s(\tilde{g} H)]
$$

we can convert every left invariant differential operator $D: \Gamma(\Sigma(G / H)) \longrightarrow \Gamma(\tilde{\Sigma}(G / H))$ of order $r \geq 0$ between sections of homogeneous vector bundles $\Sigma(G / H)$ and $\tilde{\Sigma}(G / H)$ into a left invariant section of the homogeneous vector bundle $\operatorname{Hom}\left(\operatorname{Jet}^{r} \Sigma(G / H), \tilde{\Sigma}(G / H)\right)$. According to Lemma 2.6 this left invariant total symbol section corresponds to an $H$-equivariant homomorphism $\operatorname{Jet}^{r} \Sigma \longrightarrow \tilde{\Sigma}$ between the respective representations $\operatorname{Jet}^{r} \Sigma$ and $\tilde{\Sigma}$ of $H$. In order to classify left invariant connections using this idea we need a suitable model, say

$$
\operatorname{Jet}^{1} \Sigma:=\operatorname{ker}\left(\left(\mathfrak{g}^{*} \otimes \Sigma\right) \oplus \Sigma \longrightarrow \mathfrak{h}^{*} \otimes \Sigma, \quad d s \oplus s \longmapsto \operatorname{res}_{\mathfrak{h}}(d s)+\delta s\right)
$$

for the representation $\operatorname{Jet}^{1} \Sigma$, where $(\delta s)(Z):=Z \star s$ for all $Z \in \mathfrak{h}$. This model fits nicely into the short exact sequence of representations to be expected from the symbol sequence

$$
0 \longrightarrow(\mathfrak{g} / \mathfrak{h})^{*} \otimes \Sigma \stackrel{\iota}{\longrightarrow} \mathrm{Jet}^{1} \Sigma \stackrel{\mathrm{pr}}{\longrightarrow} \Sigma \longrightarrow 0
$$

with $\iota(\sigma):=\sigma \oplus 0$ and $\operatorname{pr}(d s \oplus s):=s$. In consequence the algebraic Definition 2.3 of left invariant connections on homogeneous vector bundles is based on the following bijection

$$
\nabla(d s \oplus s)=d s+A s \quad \Longleftrightarrow \quad A s=\nabla(d s \oplus s)-d s
$$

between the $H$-equivariant sections $\nabla: \operatorname{Jet}^{1} \Sigma \longrightarrow(\mathfrak{g} / \mathfrak{h})^{*} \otimes \Sigma$ of the symbol sequence and the $H$-equivariant extensions $A: \mathfrak{g} \longrightarrow$ End $\Sigma$ of the infinitesimal representation of $\mathfrak{h}$ on $\Sigma$.

\section{Lemma 2.7 (Curvature and Torsion of a Left Invariant Connection)}

The curvature of a left invariant connection $A: \mathfrak{g} \longrightarrow$ End $\Sigma$ on a homogeneous vector bundle $\Sigma(G / H)$ is the End $\Sigma$-valued 2 -form $R \in\left[\Lambda^{2}(\mathfrak{g} / \mathfrak{h})^{*} \otimes \text { End } \Sigma\right]^{H}$ on $\mathfrak{g} / \mathfrak{h}$ defined by

$$
R_{X+\mathfrak{h}, Y+\mathfrak{h}}:=\left[A_{X}, A_{Y}\right]-A_{[X, Y]}
$$

for representatives $X, Y \in \mathfrak{g}$. In the same vein the torsion of a left invariant connection $A: \mathfrak{g} \longrightarrow$ End $\mathfrak{g} / \mathfrak{h}$ on the tangent bundle $T(G / H)$ of the homogeneous space $G / H$ is defined as the $\mathfrak{g} / \mathfrak{h}$-valued 2 -form $T \in\left[\Lambda^{2}(\mathfrak{g} / \mathfrak{h})^{*} \otimes(\mathfrak{g} / \mathfrak{h})\right]^{H}$ given on representatives $X, Y \in \mathfrak{g}$ by:

$$
T(X+\mathfrak{h}, Y+\mathfrak{h}) \equiv A_{X}(Y+\mathfrak{h})-A_{Y}(X+\mathfrak{h})-[X, Y] \bmod \mathfrak{h}
$$


The usual projection from the space of linear connections on the tangent bundle $T M$ of a manifold $M$ to the space of torsion free connections works just as well for left invariant connections on the tangent bundle of a homogeneous space $G / H$. Because the algebraic torsion $T \in\left[\Lambda^{2}(\mathfrak{g} / \mathfrak{h})^{*} \otimes(\mathfrak{g} / \mathfrak{h})\right]^{H}$ defined in Lemma 2.7 is $H$-invariant, we may use it to modify the left invariant connection $A: \mathfrak{g} \longrightarrow$ End $\mathfrak{g} / \mathfrak{h}$ to the $H$-equivariant linear map

$$
A_{X}^{\mathrm{tf}}(Y+\mathfrak{h}):=A_{X}(Y+\mathfrak{h})-\frac{1}{2} T(X+\mathfrak{h}, Y+\mathfrak{h})
$$

which still extends the infinitesimal isotropy representation $\star$ of $\mathfrak{h}$ on $\mathfrak{g} / \mathfrak{h}$. Clearly the torsion $T^{\mathrm{tf}}$ of this new left invariant connection $A^{\mathrm{tf}}: \mathfrak{g} \longrightarrow$ End $\mathfrak{g} / \mathfrak{h}$ vanishes by construction. Thinking of the torsion $T(X+\mathfrak{h}, Y+\mathfrak{h})=: T_{X+\mathfrak{h}}(Y+\mathfrak{h})$ as an endomorphism valued 1 -form on $\mathfrak{g} / \mathfrak{h}$ and using $\left(A_{X} \star T\right)_{Y+\mathfrak{h}}:=\left[A_{X}, T_{Y+\mathfrak{h}}\right]-T_{A_{X}(Y+\mathfrak{h})}$ we find for the curvature:

$$
\begin{aligned}
R_{X}^{\mathrm{tf}} & +\mathfrak{h}, Y+\mathfrak{h} \\
& =\left[A_{X}-\frac{1}{2} T_{X+\mathfrak{h}}, A_{Y}-\frac{1}{2} T_{Y+\mathfrak{h}}\right]-A_{[X, Y]}+\frac{1}{2} T_{A_{X}(Y+\mathfrak{h})-A_{Y}(X+\mathfrak{h})-T(X+\mathfrak{h}, Y+\mathfrak{h})} \\
& =R_{X+\mathfrak{h}, Y+\mathfrak{h}}-\frac{1}{2}\left(\left(A_{X} \star T\right)_{Y+\mathfrak{h}}-\left(A_{Y} \star T\right)_{X+\mathfrak{h}}\right)+\frac{1}{4}\left(\left[T_{X+\mathfrak{h}}, T_{Y+\mathfrak{h}}\right]-2 T_{T(X+\mathfrak{h}, Y+\mathfrak{h})}\right)
\end{aligned}
$$

This formula is remarkably similar to the standard formula for the curvature $R^{\mathrm{tf}}$ of the torsion free projection $\nabla^{\mathrm{tf}}:=\nabla-\frac{1}{2} T$ of a connection on the tangent bundle a manifold $M$

$$
R_{X, Y}^{\mathrm{tf}}=R_{X, Y}-\frac{1}{2}\left(\left(\nabla_{X} T\right)_{Y}-\left(\nabla_{Y} T\right)_{X}\right)+\frac{1}{4}\left(\left[T_{X}, T_{Y}\right]-2 T_{T(X, Y)}\right)
$$

provided we identify $\left(A_{X} \star T\right)_{Y+\mathfrak{h}}$ with $\left(\nabla_{X} T\right)_{Y}$. Generalizing this identification we find:

\section{Remark 2.8 (Covariant Derivatives of Left Invariant Sections)}

The covariant derivative of a left invariant section $s \in \Gamma(\Sigma(G / H))^{G}$ of a homogeneous vector bundle $\Sigma(G / H)$ under a left invariant connection $A: \mathfrak{g} \longrightarrow$ End $\Sigma$ is a left invariant section $\nabla^{A}$ s of the homogeneous vector bundle $T^{*}(G / H) \otimes \Sigma(G / H)$. In particular the identification $\Gamma(\Sigma(G / H))^{G} \cong[\Sigma]^{H}$ turns the left invariant connection into the linear map

$$
A \circledast:[\Sigma]^{H} \longrightarrow\left[(\mathfrak{g} / \mathfrak{h})^{*} \otimes \Sigma\right]^{H}, \quad s \longmapsto A \circledast s
$$

well-defined by $(A \circledast s)_{X+\mathfrak{h}}:=A_{X} s$ for $X \in \mathfrak{g}$, because $A_{X} s=X \star s=0$ for all $X \in \mathfrak{h}$.

A pleasant aspect of this description of the covariant derivative of left invariant sections is that it can be iterated with ease provided we we fix an auxiliary left invariant section $A^{\text {aux }}: \mathfrak{g} \longrightarrow$ End $\mathfrak{g} / \mathfrak{h}$ on the tangent bundle $T(G / H)$ of the homogeneous space $G / H$. The standard skew symmetrization for iterated covariant derivatives for example becomes

$$
\operatorname{alt}_{12}\left(\left(A, A^{\text {aux }}\right) \circledast(A \circledast s)\right)=R \circledast s-A_{T_{\text {aux }} \circledast s}
$$

where alt $_{12}: V^{*} \otimes\left(V^{*} \otimes \Sigma\right) \longrightarrow\left(V^{*} \otimes V^{*}\right) \otimes \Sigma$ is the skew symmetrization in the first two arguments and $T^{\text {aux }}$ the torsion of the auxiliary connection $A^{\text {aux }}$. 


\section{Formal Affine Homogeneous Spaces}

Abstracting the concept of affine homogeneous spaces developped so far into a purely algebraic concept is only possibly, if we agree to ignore the global aspects of a homogeneous space $G / H$ considered as a manifold and focus on the pair $\mathfrak{g} \supset \mathfrak{h}$ of Lie algebras instead. Since it is no longer feasible in this algebraic context to define a left invariant connection as an $H$-equivariant linear map, we are lead to define a formal affine homogeneous space as a pair $\mathfrak{g} \supset \mathfrak{h}$ of Lie algebras endowed with an $\mathfrak{h}$-equivariant extension $A: \mathfrak{g} \longrightarrow$ End $\mathfrak{g} / \mathfrak{h}$ of the infinitesimal isotropy representation $\star: \mathfrak{h} \longrightarrow$ End $\mathfrak{g} / \mathfrak{h}$. In this section we will associate two isomorphic skew algebras (End $\mathfrak{g} / \mathfrak{h}) \oplus_{\mathfrak{h}, A} \mathfrak{g} \cong($ End $\mathfrak{g} / \mathfrak{h}) \oplus_{R, T}(\mathfrak{g} / \mathfrak{h})$ to such a formal affine homogeneous space with the characteristic property that the quotient $\mathfrak{g} / \mathfrak{n}$ of $\mathfrak{g}$ by the maximal ideal $\mathfrak{n} \subset \mathfrak{h}$ contained in $\mathfrak{h}$ becomes a Lie subalgebra of these skew algebras.

\section{Definition 3.1 (Formal Affine Homogeneous Spaces)}

A formal affine homogeneous space is a pair $\mathfrak{g} \supset \mathfrak{h}$ of Lie algebras endowed with a formal left invariant connection on its isotropy representation, this is an $\mathfrak{h}$-equivariant linear map $A: \mathfrak{g} \longrightarrow$ End $\mathfrak{g} / \mathfrak{h}$ extending the isotropy representation $\star: \mathfrak{h} \longrightarrow$ End $\mathfrak{g} / \mathfrak{h}$. The curvature $R \in\left[\Lambda^{2}(\mathfrak{g} / \mathfrak{h})^{*} \otimes(\text { End } \mathfrak{g} / \mathfrak{h})\right]^{\mathfrak{h}}$ and the torsion $T \in\left[\Lambda^{2}(\mathfrak{g} / \mathfrak{h})^{*} \otimes(\mathfrak{g} / \mathfrak{h})\right]^{\mathfrak{h}}$ of a formal affine homogeneous space are defined by the same formulas as for an actual homogeneous space:

$$
\begin{array}{ll}
R_{X+\mathfrak{h}, Y+\mathfrak{h}} & :=\left[A_{X}, A_{Y}\right]-A_{[X, Y]} \\
T(X+\mathfrak{h}, Y+\mathfrak{h}) & :=A_{X}(Y+\mathfrak{h})-A_{Y}(X+\mathfrak{h})-[X, Y]+\mathfrak{h}
\end{array}
$$

Evidently every affine homogeneous space $G / H$ endowed with a left invariant connection $A: \mathfrak{g} \longrightarrow$ End $\mathfrak{g} / \mathfrak{h}$ on its tangent bundle $T(G / H)$ defines a formal affine homogeneous spaces $\mathfrak{g} \supset \mathfrak{h}$ with formal connection $A$. On the other hand we may encounter serious problems in integrating a formal affine homogeneous space to an actual homogeneous space, because the subset $\exp \mathfrak{h} \subset G$ of the simply connected Lie group $G$ with Lie algebra $\mathfrak{g}$ does not in general generate a closed subgroup $H \subset G$, quite simple and beautiful conterexamples in this direction have been constructed by Kowalski [K1].

In the case $H$ fails to be a closed subgroup of $G$ the formal affine homogeneous space $\mathfrak{g} \supset \mathfrak{h}$ does not integrate to a true affine homogeneous space $G / H$. In order to define some kind of surrogate we may consider the closure $\bar{H} \supset H$ of $H$ in $G$, which is a Lie subgroup of $G$ with its own Lie algebra $\overline{\mathfrak{h}} \supset \mathfrak{h}$. Since the adjoint action $\star: G \times \mathfrak{g} \longrightarrow \mathfrak{g}$ of the Lie group $G$ on its Lie algebra $\mathfrak{g}$ is continuous, the $H$-invariant subspace $\mathfrak{h} \subset \mathfrak{g}$ is actually $\bar{H}$-invariant so that $\overline{\mathfrak{h}} / \mathfrak{h}$ is a Lie algebra. The resulting short exact sequence of representations of $\bar{H}$

$$
0 \longrightarrow \overline{\mathfrak{h}} / \mathfrak{h} \longrightarrow \mathfrak{g} / \mathfrak{h} \longrightarrow \mathfrak{g} / \overline{\mathfrak{h}} \longrightarrow 0
$$

corresponds to a short exact sequence of homogeneous vector bundles on $G / \bar{H}$ involving the tangent bundle $T(G / \bar{H})$ modelled on $\mathfrak{g} / \overline{\mathfrak{h}}$ and the homogeneous Lie algebra bundle modelled on $\overline{\mathfrak{h}} / \mathfrak{h}$. Hence the homogeneous vector bundle on $G / \bar{H}$ modelled on $\mathfrak{g} / \mathfrak{h}$ is a transitive Lie algebroid bundle " $T(G / H)$ " endowed with a left invariant connection $\nabla$, whose curvature $R$ and torsion $T$ equal the formal curvature and torsion defined above. In this context we recall that the torsion is actually defined for linear connections on transitive Lie algebroids. 


\section{Definition 3.2 (Skew Algebra associated to Connection)}

Consider a left invariant, not necessarily torsion free connection $A: \mathfrak{g} \longrightarrow$ End $\mathfrak{g} / \mathfrak{h}$ on the isotropy representation $\mathfrak{g} / \mathfrak{h}$ of a pair $\mathfrak{g} \supset \mathfrak{h}$ of Lie algebras. The quotient of $($ End $\mathfrak{g} / \mathfrak{h}) \oplus \mathfrak{g}$

$$
(\text { End } \mathfrak{g} / \mathfrak{h}) \oplus_{\mathfrak{h}, A} \mathfrak{g}:=(\text { End } \mathfrak{g} / \mathfrak{h}) \oplus \mathfrak{g} /\{(-H \star) \oplus H \mid H \in \mathfrak{h}\}
$$

by the diagonal subspace $\mathfrak{h}$ can be endowed with a skew symmetric bilinear bracket via:

$$
\begin{aligned}
{\left[\mathfrak{X} \oplus_{\mathfrak{h}, A} X, \mathfrak{Y} \oplus_{\mathfrak{h}, A} Y\right]:=[\mathfrak{X}, \mathfrak{Y}] \oplus_{\mathfrak{h}, A}[X, Y] } & +\left(\left[\mathfrak{X}, A_{Y}\right]-A_{\mathfrak{X} Y}\right) \oplus_{\mathfrak{h}, A} \mathfrak{X} Y \\
& -\left(\left[\mathfrak{Y}, A_{X}\right]-A_{\mathfrak{Y} X}\right) \oplus_{\mathfrak{h}, A} \mathfrak{Y} X
\end{aligned}
$$

The notation (End $\mathfrak{g} / \mathfrak{h}) \oplus_{\mathfrak{h}, A} \mathfrak{g}$ reflects the dependence of the resulting skew algebra on $A$.

Some thoughts should be spent on the interpretation of the terms $\mathfrak{X} Y$ and $\mathfrak{Y} X$ in the definition of the bracket above, which are used as if the classes $\mathfrak{X}(Y+\mathfrak{h})$ and $\mathfrak{Y}(X+\mathfrak{h})$ in $\mathfrak{g} / \mathfrak{h}$ were well defined elements of $\mathfrak{g}$. Nevertheless the resulting ambiguities cancel out in the quotient $($ End $\mathfrak{g} / \mathfrak{h}) \oplus_{\mathfrak{h}, A} \mathfrak{g}$ of $($ End $\mathfrak{g} / \mathfrak{h}) \oplus \mathfrak{g}$ by the diagonal $\{(-H \star) \oplus H \mid H \in \mathfrak{h}\}$

$$
\left(\left[\mathfrak{X}, A_{Y}\right]-A_{\mathfrak{X} Y}\right) \oplus_{\mathfrak{h}, A} \mathfrak{X} Y=\left[\mathfrak{X}, A_{Y}\right] \oplus_{\mathfrak{h}, A} 0+\left(-A_{\mathfrak{X} Y}\right) \oplus_{\mathfrak{h}, A} \mathfrak{X} Y
$$

as long as we take the same representatives $\mathfrak{X} Y$ and $\mathfrak{Y} X$ in $\mathfrak{g}$ for the classes $\mathfrak{X}(Y+\mathfrak{h})$ and $\mathfrak{Y}(X+\mathfrak{h})$ in $\mathfrak{g} / \mathfrak{h}$ on both sides of $\oplus$. With this proviso the bracket of two elements in (End $\mathfrak{g} / \mathfrak{h}) \oplus \mathfrak{g}$ is well defined in the quotient $($ End $\mathfrak{g} / \mathfrak{h}) \oplus_{\mathfrak{h}, A} \mathfrak{g}$. The bracket will descend to a skew algebra structure on (End $\mathfrak{g} / \mathfrak{h}) \oplus_{\mathfrak{h}, A} \mathfrak{g}$, if all elements representing 0 in the quotient have vanishing brackets with all other elements of $($ End $\mathfrak{g} / \mathfrak{h}) \oplus \mathfrak{g}$. Observing that the classes $H \star(Y+\mathfrak{h})$ and $\mathfrak{Y}(H+\mathfrak{h})$ for given $H \in \mathfrak{h}$ are represented in $\mathfrak{g}$ by $[H, Y]$ and 0 we find

$$
\begin{aligned}
& {[(-H \star) \oplus H, \mathfrak{Y} \oplus Y]} \\
& \quad=[-H \star, \mathfrak{Y}] \oplus_{\mathfrak{h}, A}[H, Y]+\left(\left[-H \star, A_{Y}\right]+A_{[H, Y]}-\left[\mathfrak{Y}, A_{H}\right]\right) \oplus_{\mathfrak{h}, A}(-[H, Y]) \\
& \quad=-\left(\left[H \star, A_{Y}\right]-A_{[H, Y]}\right) \oplus_{\mathfrak{h}, A} 0
\end{aligned}
$$

using $A_{H}=H \star$ in the second line. Due to the characteristic infinitesimal $\mathfrak{h}$-equivariance of the left invariant connection $A: \mathfrak{g} \longrightarrow$ End $\mathfrak{g} / \mathfrak{h}$ the right hand side vanishes so that the bracket is in fact well-defined on the quotient $($ End $\mathfrak{g} / \mathfrak{h}) \oplus_{\mathfrak{h}, A} \mathfrak{g}$.

The inclusion of the first summand $\mathfrak{X} \longmapsto \mathfrak{X} \oplus_{\mathfrak{h}, A} 0$ is evidently an injective skew algebra homomorphism turning End $\mathfrak{g} / \mathfrak{h}$ into a Lie subalgebra of (End $\mathfrak{g} / \mathfrak{h}) \oplus_{\mathfrak{h}, A} \mathfrak{g}$. Things are slightly more complicated for the inclusion $X \longmapsto 0 \oplus_{\mathfrak{h}, A} X$ of the second summand, which may well fail to be injective. Its kernel however agrees with the kernel of the isotropy representation $\star: \mathfrak{h} \longrightarrow$ End $\mathfrak{g} / \mathfrak{h}, H \longmapsto H \star$, which in turn agrees with the maximal ideal $\mathfrak{n} \subset \mathfrak{h}$ of $\mathfrak{g}$ contained in $\mathfrak{h}$ according to Lemma 2.5. In this way the skew algebra (End $\mathfrak{g} / \mathfrak{h}$ ) $\oplus_{\mathfrak{h}, A} \mathfrak{g}$ is generated as a vector space by its two Lie subalgebras End $\mathfrak{g} / \mathfrak{h}$ and $\mathfrak{g} / \mathfrak{n}$, nevertheless the Jacobi identity will not hold true for arbitrary elements of $($ End $\mathfrak{g} / \mathfrak{h}) \oplus_{\mathfrak{h}, A} \mathfrak{g}$. 


\section{Definition 3.3 (Skew Algebra associated to Curvature-Torsion)}

Consider a left invariant connection $A: \mathfrak{g} \longrightarrow$ End $\mathfrak{g} / \mathfrak{h}$ on the isotropy representation $\mathfrak{g} / \mathfrak{h}$ of a pair $\mathfrak{g} \supset \mathfrak{h}$ of Lie algebras with associated curvature $R \in\left[\Lambda^{2}(\mathfrak{g} / \mathfrak{h})^{*} \otimes \text { End } \mathfrak{g} / \mathfrak{h}\right]^{\mathfrak{h}}$ and torsion $T \in\left[\Lambda^{2}(\mathfrak{g} / \mathfrak{h})^{*} \otimes \mathfrak{g} / \mathfrak{h}\right]^{\mathfrak{h}}$. The direct sum $($ End $\mathfrak{g} / \mathfrak{h}) \oplus(\mathfrak{g} / \mathfrak{h})$ of vector spaces is actually a skew algebra denoted by $($ End $\mathfrak{g} / \mathfrak{h}) \oplus_{R, T}(\mathfrak{g} / \mathfrak{h})$ under the following skew bilinear bracket:

$$
\left[\mathfrak{X} \oplus_{R, T} x, \mathfrak{Y} \oplus_{R, T} y\right]:=\left([\mathfrak{X}, \mathfrak{Y}]-R_{x, y}\right) \oplus_{R, T}(\mathfrak{X} y-\mathfrak{Y} x-T(x, y))
$$

Up to the additional terms $-R_{x, y}$ and $-T(x, y)$ the skew bracket on $($ End $\mathfrak{g} / \mathfrak{h}) \oplus_{R, T}(\mathfrak{g} / \mathfrak{h})$ agrees with the Lie bracket on the semidirect product (End $\mathfrak{g} / \mathfrak{h}) \oplus(\mathfrak{g} / \mathfrak{h})$ of the Lie algebra End $\mathfrak{g} / \mathfrak{h}$ with its representation $\mathfrak{g} / \mathfrak{h}$. The possible failure of the Jacobi identity for the bracket on (End $\mathfrak{g} / \mathfrak{h}) \oplus_{R, T}(\mathfrak{g} / \mathfrak{h})$ is thus due to these two additional terms, for the moment however we postpone a detailed analysis of this problem to Section 4.

\section{Lemma 3.4 (Skew Algebra Isomorphism)}

Let $A: \mathfrak{g} \longrightarrow$ End $(\mathfrak{g} / \mathfrak{h})$ be a left invariant connection on the isotropy representation $\mathfrak{g} / \mathfrak{h}$ of a pair $\mathfrak{g} \supset \mathfrak{h}$ of Lie algebras with curvature $R \in\left[\Lambda^{2}(\mathfrak{g} / \mathfrak{h})^{*} \otimes(\text { End } \mathfrak{g} / \mathfrak{h})\right]^{\mathfrak{h}}$ and torsion $T \in\left[\Lambda^{2}(\mathfrak{g} / \mathfrak{h})^{*} \otimes(\mathfrak{g} / \mathfrak{h})\right]^{\mathfrak{h}}$. The connection A defines a canonical isomorphism of skew algebras

$$
\begin{aligned}
\Phi_{A}: \quad(\text { End } \mathfrak{g} / \mathfrak{h}) \oplus_{\mathfrak{h}, A} \mathfrak{g} & \stackrel{\cong}{\longrightarrow}(\text { End } \mathfrak{g} / \mathfrak{h}) \oplus_{R, T}(\mathfrak{g} / \mathfrak{h}) \\
\mathfrak{X} \oplus_{\mathfrak{h}, A} X & \longmapsto\left(\mathfrak{X}+A_{X}\right) \oplus_{R, T}(X+\mathfrak{h})
\end{aligned}
$$

between the skew algebras associated to $A$ and $R, T$. Composing $\Phi_{A}$ with the skew algebra homomorphism $\mathfrak{g} \longrightarrow($ End $\mathfrak{g} / \mathfrak{h}) \oplus_{\mathfrak{h}, A} \mathfrak{g}, X \longmapsto 0 \oplus_{\mathfrak{h}, A} X$, we may identify the quotient $\mathfrak{g} / \mathfrak{n}$ of $\mathfrak{g}$ by the maximal ideal $\mathfrak{n} \subset \mathfrak{g}$ contained in $\mathfrak{h}$ with a Lie subalgebra of $($ End $\mathfrak{g} / \mathfrak{h}) \oplus_{R, T}(\mathfrak{g} / \mathfrak{h})$ :

$$
\mathfrak{g} / \mathfrak{n} \stackrel{\subset}{\longrightarrow}(\text { End } \mathfrak{g} / \mathfrak{h}) \oplus_{R, T}(\mathfrak{g} / \mathfrak{h}), \quad X+\mathfrak{n} \longmapsto A_{X} \oplus_{R, T}(X+\mathfrak{h})
$$

Proof: A short inspection shows that $\Phi_{A}$ is well defined on the quotient (End $\left.\mathfrak{g} / \mathfrak{h}\right) \oplus_{\mathfrak{h}, A} \mathfrak{g}$ due to $A_{H}=H \star$ for all $H \in \mathfrak{h}$. Moreover $\Phi_{A}$ is a linear isomorphism with well-defined inverse $\Phi_{A}^{-1}:($ End $\mathfrak{g} / \mathfrak{h}) \oplus_{R, T}(\mathfrak{g} / \mathfrak{h}) \longrightarrow($ End $\mathfrak{g} / \mathfrak{h}) \oplus_{\mathfrak{h}, A} \mathfrak{g}$ given explicitly by

$$
\Phi_{A}^{-1}\left(\mathfrak{X} \oplus_{R, T} x\right):=\left(\mathfrak{X}-A_{X}\right) \oplus_{\mathfrak{h}, A} X
$$

where $X \in \mathfrak{g}$ represents the class $x \in \mathfrak{g} / \mathfrak{h}$. Eventually $\Phi_{A}$ is a homomorphism of algebras

$$
\begin{aligned}
{\left[\Phi_{A}\left(\mathfrak{X} \oplus_{\mathfrak{h}, A} X\right), \Phi_{A}\left(\mathfrak{Y} \oplus_{\mathfrak{h}, A} Y\right)\right] } \\
=\left([\mathfrak{X}, \mathfrak{Y}]+\left[A_{X}, \mathfrak{Y}\right]+\left[\mathfrak{X}, A_{Y}\right]+\left[A_{X}, A_{Y}\right]-R_{X+\mathfrak{h}, Y+\mathfrak{h}}\right) \\
\quad \oplus_{R, T}\left(\left(\mathfrak{X}+A_{X}\right)(Y+\mathfrak{h})-\left(\mathfrak{Y}+A_{Y}\right)(X+\mathfrak{h})-T(X+\mathfrak{h}, Y+\mathfrak{h})\right) \\
=\left([\mathfrak{X}, \mathfrak{Y}]+\left[\mathfrak{X}, A_{Y}\right]-\left[\mathfrak{Y}, A_{X}\right]+A_{[X, Y]}\right) \oplus_{R, T}(\mathfrak{X} Y-\mathfrak{Y} X-[X, Y]+\mathfrak{h}) \\
=\Phi_{A}\left(\left([\mathfrak{X}, \mathfrak{Y}]+\left[\mathfrak{X}, A_{Y}\right]-A_{\mathfrak{X} Y}-\left[\mathfrak{Y}, A_{X}\right]+A_{\mathfrak{Y} X}\right) \oplus_{\mathfrak{h}, A}(\mathfrak{X} Y-\mathfrak{Y} X+[X, Y])\right)
\end{aligned}
$$

where $\mathfrak{X} Y, \mathfrak{Y} X \in \mathfrak{g}$ denote fixed representatives for the corresponding classes in $\mathfrak{g} / \mathfrak{h}$. 


\section{A Parametrization of Formal Affine Spaces}

In this section we change our point of view completely away from a fixed formal affine homogeneous space towards a parametrization of such spaces by connection-curvature-torsion triples. In order to compare different affine homogeneous spaces we choose a linear isomorphism or frame $F: V \longrightarrow \mathfrak{g} / \mathfrak{h}$ to pull back curvature and torsion to $V$ and choose a section rep $: \mathfrak{g} / \mathfrak{h} \longrightarrow \mathfrak{g}$ to complement the information contained in $R \in \Lambda^{2} V^{*} \otimes$ End $V$ and $T \in \Lambda^{2} V^{*} \otimes V$ by an additional $A \in V^{*} \otimes$ End $V$ describing the connection. A generic triple $(A, R, T)$ of this form will certainly not come from a formal affine homogeneous space $\mathfrak{g} \supset \mathfrak{h}$, the algebraic equations characterizing connection-curvature-torsion triples $(A, R, T)$ of formal affine homogeneous spaces will be made explicit at the end of this section.

For the time being let us fix a finite dimensional vector space $V$. Augmenting a formal affine homogeneous space $\mathfrak{g} \supset \mathfrak{h}$ of dimension $\operatorname{dim} \mathfrak{g} / \mathfrak{h}=\operatorname{dim} V$ with a linear isomorphism or frame $F: V \longrightarrow \mathfrak{g} / \mathfrak{h}$ allows us to think of curvature and torsion of the left invariant connection $A: \mathfrak{g} \longrightarrow$ End $\mathfrak{g} / \mathfrak{h}$ as elements $R \in \Lambda^{2} V^{*} \otimes$ End $V$ and $T \in \Lambda^{2} V^{*} \otimes V$. Nevertheless the resulting curvature-torsion tuple $(R, T)$ does not describe the original formal affine homogeneous space completely. For this reason we choose in addition a section rep $: \mathfrak{g} / \mathfrak{h} \longrightarrow \mathfrak{g}$ of the canonical projection to $\mathfrak{g} / \mathfrak{h}$ in order to capture the information contained in the connection $A$ in a linear map $A \circ$ rep $: \mathfrak{g} / \mathfrak{h} \longrightarrow$ End $\mathfrak{g} / \mathfrak{h}$, which becomes under $F$ the End $V$-valued 1 -form $A \in V^{*} \otimes$ End $V$ on $V$ still called connection:

Definition 4.1 (Connection-Curvature-Torsion Triples)

A connection-curvature-torsion triple on a vector space $V$ is a triple of the form:

$$
(A, R, T) \in\left(V^{*} \otimes \text { End } V\right) \times\left(\Lambda^{2} V^{*} \otimes \text { End } V\right) \times\left(\Lambda^{2} V^{*} \otimes V\right)
$$

Every such a triple $(A, R, T)$ endows End $V \oplus_{R, T} V:=$ End $V \oplus V$ with the skew bracket:

$$
\left[\mathfrak{X} \oplus_{R, T} x, \mathfrak{Y} \oplus_{R, T} y\right]:=\left([\mathfrak{X}, \mathfrak{Y}]-R_{x, y}\right) \oplus_{R, T}(\mathfrak{X} y-\mathfrak{Y} x-T(x, y))
$$

The connection-curvature-torsion triples $(A, R, T)$ coming from actual formal affine homogeneous spaces $\mathfrak{g} \supset \mathfrak{h}$ augmented by frames $F: V \longrightarrow \mathfrak{g} / \mathfrak{h}$ and sections rep $: \mathfrak{g} / \mathfrak{h} \longrightarrow \mathfrak{g}$ are characterized by the fact that the skew algebra End $V \oplus_{R, T} V$ contains a Lie subalgebra

$$
\mathfrak{g} / \mathfrak{n} \stackrel{\subset}{\longrightarrow}(\text { End } \mathfrak{g} / \mathfrak{h}) \oplus_{R, T}(\mathfrak{g} / \mathfrak{h}) \stackrel{\cong}{\longrightarrow} \text { End } V \oplus_{R, T} V
$$

isomorphic to $\mathfrak{g} / \mathfrak{n}$ according to Lemma 3.4 , which contains the image of the extension of $A$

$$
A^{\text {ext }}: \quad V \longrightarrow \text { End } V \oplus_{R, T} V, \quad x \longmapsto A_{x} \oplus_{R, T} x
$$

and thus projects onto $V$ under the canonical projection End $V \oplus_{R, T} V \longrightarrow V$. Conversely: 


\section{Definition 4.2 (Isotropy Algebra and Tautological Connection)}

Consider a Lie subalgebra $\mathfrak{g}$ of the skew algebra End $V \oplus_{R, T} V$ associated to a curvaturetorsion tuple $(R, T) \in \Lambda^{2} V^{*} \otimes$ End $V \times \Lambda^{2} V^{*} \otimes V$, which projects surjectively onto $V$ under:

$$
\text { End } V \oplus_{R, T} V \longrightarrow V, \quad \mathfrak{X} \oplus_{R, T} x \longmapsto x
$$

Defining the isotropy algebra $\mathfrak{h}:=\mathfrak{g} \cap$ End $V$ as the kernel of this projection we observe that the canonical projection becomes an isomorphism $\mathfrak{g} / \mathfrak{h} \stackrel{\cong}{\longrightarrow} V$ of the isotropy representation of the pair $\mathfrak{g} \supset \mathfrak{h}$ with $V$, with this proviso the canonical projection to the first summand

$$
A^{\text {taut }}: \quad \text { End } V \oplus_{R, T} V \longrightarrow \text { End } V, \quad \mathfrak{X} \oplus_{R, T} x \longmapsto \mathfrak{X}
$$

becomes the tautological left invariant connection $A^{\text {taut }}: \mathfrak{g} \longrightarrow$ End $V$ on $V \cong \mathfrak{g} / \mathfrak{h}$.

Although or perhaps because all arguments and calculations involving the tautological connection $A^{\text {taut }}$ eventually reduce to tautologies it is difficult and slightly confusing to use it in explicit calculations. Nevertheless the tautological connection $A^{\text {taut }}$ is $\mathfrak{h}$-equivariant to due $\left[\mathfrak{X} \oplus_{R, T} 0, \mathfrak{Y} \oplus_{R, T} y\right]=[\mathfrak{X}, \mathfrak{Y}] \oplus_{R, T} \mathfrak{X} y$ and its curvature agrees with $R \in \Lambda^{2} V^{*} \otimes$ End $V$ :

$$
\left[A_{\mathfrak{X} \oplus_{R, T}}^{\text {taut }}, A_{\mathfrak{Y} \oplus_{R, T} y}^{\text {taut }}\right]-A_{\left[\mathfrak{X} \oplus_{R, T} x, \mathfrak{Y} \oplus_{R, T} y\right]}^{\text {taut }}=[\mathfrak{X}, \mathfrak{Y}]-\left([\mathfrak{X}, \mathfrak{Y}]-R_{x, y}\right)=R_{x, y}
$$

A very similar argument can be made to verify that the torsion of the tautological connection agrees with $T \in \Lambda^{2} V^{*} \otimes V$. In consequence every Lie subalgebra $\mathfrak{g} \subset$ End $V \oplus_{R, T} V$ projecting surjectively onto $V$ defines a formal affine homogeneous space $\mathfrak{g} \supset \mathfrak{h}$ with tautological frame $F: V \longrightarrow \mathfrak{g} / \mathfrak{h}$ under the tautological left invariant connection $A^{\text {taut }}: \mathfrak{g} \longrightarrow$ End $V$. Motivated by Lemma 2.8 we now define for every representation $\Sigma$ of the Lie algebra End $V$ and every $d \geq 0$ the bilinear operation

$$
\circledast: \quad \otimes^{d} V^{*} \otimes \text { End } V \times \otimes^{\bullet} V^{*} \otimes \Sigma \longrightarrow \otimes^{\bullet+d} V^{*} \otimes \Sigma
$$

by setting

$$
(Q \circledast s)\left(x_{1}, \ldots, x_{d} ; y_{1}, y_{2}, \ldots\right):=\left(Q_{x_{1}, \ldots, x_{d}} \star s\right)\left(y_{1}, y_{2}, \ldots\right)
$$

where $\star$ denotes the tensor product representation of End $V$ on $\otimes^{\bullet} V^{*} \otimes \Sigma$.

\section{Definition 4.3 (Formal Covariant Derivatives of $R$ and $T$ )}

For a given connection-curvature-torsion triple $(A, R, T)$ on a finite-dimensional vector space $V$ we define the formal iterated covariant derivatives of $R$ and $T$ by setting:

$$
\begin{aligned}
& \nabla^{r} T:=\underbrace{A \circledast(A \circledast(\ldots(A}_{r \text { times }} \circledast T) \ldots)) \in \otimes^{r} V^{*} \otimes\left(\Lambda^{2} V^{*} \otimes V\right) \\
& \nabla^{r} R:=\underbrace{A \circledast(A \circledast(\ldots(A}_{r \text { times }} \circledast R) \ldots)) \in \otimes^{r} V^{*} \otimes\left(\Lambda^{2} V^{*} \otimes \text { End } V\right)
\end{aligned}
$$




\section{Definition 4.4 (Stabilizer Filtration)}

Associated to every connection-curvature torsion triple is the strictly descending filtration

$$
\text { End } V=\ldots=\mathfrak{h}_{-2}=\mathfrak{h}_{-1} \supsetneq \mathfrak{h}_{0} \supsetneq \ldots \supsetneq \mathfrak{h}_{s-1} \supsetneq \mathfrak{h}_{s}=\mathfrak{h}_{s+1}=\ldots=\mathfrak{h}_{\infty}
$$

of End $V$ defined recursively by $\mathfrak{h}_{0}:=\mathfrak{s t a b} R \cap \mathfrak{s t a b} T \subset$ End $V$ and for all $r \geq 0$ :

$$
\mathfrak{h}_{r+1}:=\left\{\mathfrak{X} \in \mathfrak{h}_{r} \mid\left[\mathfrak{X}, A_{x}\right] \equiv A_{\mathfrak{X} x} \bmod \mathfrak{h}_{r} \text { for all } x \in V\right\}
$$

The minimal $s \geq-1$ with equality $\mathfrak{h}_{s}=\mathfrak{h}_{s+1}$ is called the Singer invariant of $(A, R, T)$.

By its recursive definition this filtration is strictly descending in the sense that it becomes stationary at the first equality $\mathfrak{h}_{s}=\mathfrak{h}_{s+1}$ of successive filtration steps for some $s \geq 0$, in passing we observe that this argument works even for $s=-1$, because the equality $\mathfrak{h}_{-1}=\mathfrak{h}_{0}$ renders the stipulated congruences $\left[\mathfrak{X}, A_{x}\right] \equiv A_{\mathfrak{X} x}$ modulo End $V$ trivial. A better way to understand the recursive definition of the stabilizer filtration is to observe that for every subalgebra $\hat{\mathfrak{h}} \subset$ End $V$ the vector space $V^{*} \otimes($ End $V / \hat{\mathfrak{h}})$ is actually a representation of $\hat{\mathfrak{h}}$ :

$$
(\mathfrak{X} \star A)_{x}:=\mathfrak{X} \star A_{x}-A_{\mathfrak{X} \star x}+\hat{\mathfrak{h}}=\left[\mathfrak{X}, A_{x}\right]-A_{\mathfrak{X} x}+\hat{\mathfrak{h}}
$$

By induction it thus follows that all subspaces $\mathfrak{h}_{r} \subset$ End $V$ of the stabilizer filtration are subalgebras, in fact this is true by definition for $\mathfrak{h}_{0}:=\mathfrak{s t a b} R \cap \mathfrak{s t a b} T$, whereas $\mathfrak{h}_{r+1}$ for $r \geq 0$ is the stabilizer subalgebra of the class $A+V^{*} \otimes \mathfrak{h}_{r}$ represented by $A$ in the $\mathfrak{h}_{r}-$ representation $V^{*} \otimes\left(\right.$ End $\left.V / \mathfrak{h}_{r}\right)$. A remarkable consequence of this observation is the following very neat interpretation of the stabilizer filtration in terms of covariant derivatives:

\section{Lemma 4.5 (Interpretation of the Stabilizer Filtration)}

The strictly descending filtration $\mathfrak{h}$. of End $V$ associated to a connection-curvature-torsion triple $(A, R, T)$ can be interpreted geometrically as the filtration given by the joint stabilizers

$$
\mathfrak{h}_{r}=\mathfrak{s t a b}\left(R \oplus \nabla R \oplus \ldots \oplus \nabla^{r} R\right) \cap \mathfrak{s t a b}\left(T \oplus \nabla T \oplus \ldots \oplus \nabla^{r} T\right)
$$

of the iterated covariant derivatives of curvature $R$ and torsion $T$ up to order $r \geq 0$.

Actually this Lemma is a special case of a more general fact, for every representation $\Sigma$ of the Lie algebra End $V$ and every $s \in \Sigma$ with stabilizer $\mathfrak{s t a b} s \subset$ End $V$ the linear map

$$
V^{*} \otimes(\text { End } V / \mathfrak{s t a b} s) \longrightarrow V^{*} \otimes \Sigma, \quad A \longmapsto A \circledast s
$$

defined by $(A \circledast s)_{x}:=A_{x} \star s$ is well-defined, injective and equivariant under $\mathfrak{s t a b} s$. Since the bilinear operation $\circledast$ is naturally defined and thus equivariant under End $V$ in the sense

$$
\mathfrak{X} \star(A \circledast s)=(\mathfrak{X} \star A) \circledast s+A \circledast(\mathfrak{X} \star s)
$$

for $\mathfrak{X} \in$ End $V$ we find that $\mathfrak{X} \in \mathfrak{s t a b} s$ stabilizes $A \in V^{*} \otimes($ End $V / \mathfrak{s t a b} s)$, if and only if:

$$
(\mathfrak{X} \star A) \circledast s=0=\mathfrak{X} \star(A \circledast s)
$$


Using this general argument the statement of the Lemma follows by an easy induction based on the definition $\mathfrak{h}_{0}:=\mathfrak{s t a b} R \cap \mathfrak{s t a b} T$ as well as on the recursive definition of $\mathfrak{h}_{r+1}$ as the stabilizer of the connection class $A+V^{*} \otimes \mathfrak{h}_{r} \in V^{*} \otimes\left(\right.$ End $\left.V / \mathfrak{h}_{r}\right)$.

For a little interlude in our algebraic considerations let us now recall the definition of the twisted exterior derivative associated to a connection $\nabla$ on a vector bundle $\Sigma M$ over a smooth manifold $M$. The twisted exterior derivative $d^{\nabla}$ is a first order differential operator

$$
d^{\nabla}: \quad \Gamma\left(\Lambda^{\bullet} T^{*} M \otimes \Sigma M\right) \longrightarrow \Gamma\left(\Lambda^{\bullet+1} T^{*} M \otimes \Sigma M\right), \quad \omega \longmapsto d^{\nabla} \omega
$$

on the differential forms on $M$ with values in $\Sigma M$ defined on a $\Sigma M$-valued $r$-form $\omega$ by:

$$
\begin{aligned}
\left(d^{\nabla} \omega\right)\left(X_{0}, \ldots, X_{r}\right):= & \sum_{\mu=0}^{r}(-1)^{\mu} \nabla_{X_{\mu}}\left(\omega\left(X_{0}, \ldots, \widehat{X}_{\mu}, \ldots, X_{r}\right)\right) \\
& -\sum_{0 \leq \mu<\nu \leq r}(-1)^{\mu+\nu-1} \omega\left(\left[X_{\mu}, X_{\nu}\right], X_{0}, \ldots, \widehat{X}_{\mu}, \ldots, \widehat{X}_{\nu}, \ldots, X_{r}\right)
\end{aligned}
$$

A classical formula tells us that with an auxiliary connection $\nabla^{\text {aux }}$ on $T M$ we may write

$$
\begin{aligned}
d^{\nabla} \omega\left(X_{0}, \ldots, X_{r}\right)= & \sum_{\mu=0}^{r}(-1)^{\mu}\left(\left(\nabla, \nabla^{\mathrm{aux}}\right)_{X_{\mu}} \omega\right)\left(X_{0}, \ldots, \widehat{X}_{\mu}, \ldots, X_{r}\right) \\
& +\sum_{0 \leq \mu<\nu \leq r}(-1)^{\mu+\nu-1} \omega\left(T^{\mathrm{aux}}\left(X_{\mu}, X_{\nu}\right), X_{0}, \ldots, \widehat{X}_{\mu}, \ldots, \widehat{X}_{\nu}, \ldots, X_{r}\right)
\end{aligned}
$$

where $T^{\text {aux }} \in \Gamma\left(\Lambda^{2} T^{*} M \otimes T M\right)$ is the torsion of the auxiliary connection $\nabla^{\text {aux }}$ and:

$$
\begin{aligned}
\left(\left(\nabla, \nabla^{\text {aux }}\right)_{X} \omega\right)\left(X_{1}, \ldots, X_{r}\right) & \\
& :=\nabla_{X}\left(\omega\left(X_{1}, \ldots, X_{r}\right)\right)-\sum_{\nu=1}^{r} \omega\left(X_{1}, \ldots, \nabla_{X}^{\operatorname{aux}} X_{\mu}, \ldots, X_{r}\right)
\end{aligned}
$$

In terms of twisted exterior derivatives the First and Second Bianchi Identity can be written

$$
d^{\nabla^{\text {aux }}} T^{\text {aux }}=R^{\text {aux }} \wedge \text { id } \quad d^{\nabla} R=0
$$

respectively, where $R$ and $R^{\text {aux }}$ denote the curvatures of $\nabla$ and $\nabla^{\text {aux }}$, whereas:

$$
\left(R^{\text {aux }} \wedge \text { id }\right)(X, Y, Z):=R_{X, Y}^{\operatorname{aux}} Z+R_{Y, Z}^{\operatorname{aux}} X+R_{Z, X}^{\text {aux }} Y
$$

Coming back to formal affine homogeneous spaces and the associated connection-curvaturetorsion triples $(A, R, T)$ we take the classical formula expressing $d^{\nabla}$ in terms of the connection $\left(\nabla, \nabla^{\text {aux }}\right)$ and the torsion $T^{\text {aux }}$ as a lead to define the twisted exterior derivative $d^{(A, T)}$ by

$$
\begin{aligned}
\left(d^{(A, T)} \omega\right)\left(x_{0}, \ldots, x_{r}\right):= & \sum_{\mu=0}^{r}(-1)^{\mu}\left(A_{x_{\mu}} \star \omega\right)\left(x_{0}, \ldots, \widehat{x}_{\mu}, \ldots, x_{r}\right) \\
& +\sum_{0 \leq \mu<\nu \leq r}(-1)^{\mu+\nu-1} \omega\left(T\left(x_{\mu}, x_{\nu}\right), x_{0}, \ldots, \widehat{x}_{\mu}, \ldots, \widehat{x}_{\nu}, \ldots, x_{r}\right)
\end{aligned}
$$


for every $r$-form $\omega \in \Lambda^{r} V^{*} \otimes \Sigma$ with values in a representation $\Sigma$ of the Lie algebra End $V$. Specifically for $R \in \Lambda^{2} V^{*} \otimes$ End $V$ the twisted exterior derivative reads

$$
\left(d^{(A, T)} R\right)_{x, y, z}=\left(\left(A_{x} \star R\right)_{y, z}+R_{T(y, z), x}\right)+\text { cyclic permutations of } x, y, z
$$

and an almost identical formula is valid for $d^{(A, T)} T \in \Lambda^{3} V^{*} \otimes V$. Last but not least we define the $V$-valued 3-form $R \wedge$ id $\in \Lambda^{3} V^{*} \otimes V$ by $(R \wedge$ id $)(x, y, z):=R_{x, y} z+R_{y, z} x+R_{z, x} y$.

\section{Definition 4.6 (Approximate Curvature)}

The approximate curvature tensor of a connection-curvature-torsion triple $(A, R, T)$ on a vector space $V$ is defined as an End $V$-valued 2 -form $Q(A, T) \in \Lambda^{2} V^{*} \otimes$ End $V$ on $V$ by:

$$
Q(A, T)_{x, y}:=\left[A_{x}, A_{y}\right]-A_{A_{x} y-A_{y} x-T(x, y)}
$$

In order to study the failure of the Jacobi identity for the bracket of the skew algebra End $V \oplus_{R, T} V$ associated to a connection-curvature-torsion triple $(A, R, T)$ we consider the trilinear standard Jacobiator defined as an alternating 3 -form Jac on End $V \oplus_{R, T} V$ by:

$$
\begin{aligned}
\operatorname{Jac}\left(\mathfrak{X} \oplus_{R, T} x, \mathfrak{Y} \oplus_{R, T}, \mathfrak{Z} \oplus_{R, T} z\right):= & +\left[\mathfrak{X} \oplus_{R, T} x,\left[\mathfrak{Y} \oplus_{R, T} y, \mathfrak{Z} \oplus_{R, T} z\right]\right] \\
& +\left[\mathfrak{Y} \oplus_{R, T} y,\left[\mathfrak{Z} \oplus_{R, T} z, \mathfrak{X} \oplus_{R, T} x\right]\right] \\
& +\left[\mathfrak{Z} \oplus_{R, T} z,\left[\mathfrak{X} \oplus_{R, T} x, \mathfrak{Y} \oplus_{R, T} y\right]\right]
\end{aligned}
$$

Observing that the bracket with $\mathfrak{X} \in$ End $V$ reproduces the infinitesimal representation

$$
\left[\mathfrak{X} \oplus_{R, T} 0, \mathfrak{Y} \oplus_{R, T} y\right]=[\mathfrak{X}, \mathfrak{Y}] \oplus_{R, T} \mathfrak{X} y=\mathfrak{X} \star\left(\mathfrak{Y} \oplus_{R, T} y\right)
$$

of End $V$ on End $V \oplus_{R, T} V$ we may calculate the Jacobiator for the special choice

$$
\begin{aligned}
\operatorname{Jac}\left(\mathfrak{X} \oplus_{R, T} 0,0 \oplus_{R, T} y, 0 \oplus_{R, T} z\right) \\
=\left[\mathfrak{X} \oplus_{R, T} 0,\left(-R_{x, y}\right) \oplus_{R, T}(-T(x, y))\right] \\
\quad-\left[0 \oplus_{R, T} y, 0 \oplus_{R, T} \mathfrak{X} z\right]+\left[0 \oplus_{R, T} z, 0 \oplus_{R, T} \mathfrak{X} y\right] \\
=\quad\left(-\left[\mathfrak{X}, R_{y, z}\right]+R_{\mathfrak{X} y, z}+R_{y, \mathfrak{X} z}\right) \oplus_{R, T}(-\mathfrak{X} T(y, z)+T(\mathfrak{X} y, z)+T(y, \mathfrak{X} z)) \\
=\left(-(\mathfrak{X} \star R)_{y, z}\right) \oplus_{R, T}(-(\mathfrak{X} \star T)(y, z))
\end{aligned}
$$

of arguments $\mathfrak{X} \in$ End $V$ and $y, z \in V$. Similarly we obtain for all three arguments in $V$ :

$$
\begin{aligned}
\operatorname{Jac} & \left(0 \oplus_{R, T} x, 0 \oplus_{R, T} y, 0 \oplus_{R, T} z\right) \\
= & {\left[0 \oplus_{R, T} x,\left(-R_{y, z}\right) \oplus_{R, T}(-T(y, z))\right]+\text { cyclic permutations of } x, y, z } \\
= & R_{x, T(y, z)} \oplus_{R, T}\left(R_{y, z} x+T(x, T(y, z))\right)+\text { cyclic permutations of } x, y, z \\
= & \left(-d^{(0, T)} R\right)_{x, y, z} \oplus_{R, T}\left(R \wedge \mathrm{id}-d^{(0, T)} T\right)(x, y, z)
\end{aligned}
$$

The latter two results feature prominantly in the proof of the following lemma: 


\section{Lemma 4.7 (Lie Subalgebras of End $V \oplus_{R, T} V$ )}

The skew algebra End $V \oplus_{R, T} V$ associated to a connection-curvature-torsion triple $(A, R, T)$ on a vector space $V$ allows no Lie subalgebra $\mathfrak{g} \supset\left\{A_{x} \oplus_{R, T} x \mid x \in V\right\}$ unless the connection-curvature-torsion triple $(A, R, T)$ satisfies the First and Second Bianchi Identity:

$$
d^{(A, T)} T=R \wedge \mathrm{id} \quad d^{(A, T)} R=0
$$

In case the First and Second Bianchi Identity are both satisfied the isotropy algebra association $\mathfrak{g} \longmapsto \mathfrak{g} \cap$ End $V$ induces a bijection between the Lie subalgebras $\mathfrak{g} \subset$ End $V \oplus_{R, T} V$ containing $\left\{A_{x} \oplus_{R, T} x \mid x \in V\right\}$ and Lie subalgebras $\mathfrak{h} \subset$ End $V$ satisfying

$$
\mathfrak{h} \subset \mathfrak{s t a b} R \cap \mathfrak{s t a b} T \quad \mathfrak{h} \star A \subset V^{*} \otimes \mathfrak{h} \quad Q(A, T) \equiv R \bmod \Lambda^{2} V^{*} \otimes \mathfrak{h}
$$

where $\mathfrak{h} \star A \subset V^{*} \otimes \mathfrak{h}$ is a shorthand for $\left[\mathfrak{X}, A_{x}\right]-A_{\mathfrak{X} x} \equiv 0$ modulo $\mathfrak{h}$ for $x \in V, \mathfrak{X} \in \mathfrak{h}$.

Proof: Every Lie subalgebra $\mathfrak{g} \subset$ End $V \oplus_{R, T} V$ containing $\left\{A_{x} \oplus_{R, T} x \mid x \in V\right\}$ is certainly determined by its isotropy algebra $\mathfrak{h}=\mathfrak{g} \cap$ End $V$. Conversely suppose that $\mathfrak{h} \subset$ End $V$ is the isotropy algebra of the Lie subalgebra $\mathfrak{g}$ of End $V \oplus_{R, T} V$ given by:

$$
\mathfrak{g}:=\mathfrak{h}+\operatorname{span}\left\{A_{x} \oplus_{R, T} x \mid x \in V\right\}
$$

In particular then $\mathfrak{g}$ is closed under the skew bracket on End $V \oplus_{R, T} V$ so that

$$
\left[\mathfrak{X} \oplus_{R, T} 0, A_{x} \oplus_{R, T} x\right]=\left(\left[\mathfrak{X}, A_{x}\right]-A_{\mathfrak{X} x}\right) \oplus_{R, T} 0+A_{\mathfrak{X} x} \oplus_{R, T} \mathfrak{X} x
$$

is necessarily an element of $\mathfrak{g}$ proving the congruence $\left[\mathfrak{X}, A_{x}\right]-A_{\mathfrak{X} x} \equiv 0$ modulo $\mathfrak{h}$ for all $\mathfrak{X} \in \mathfrak{h}$ and $x \in V$. Similarly the result of the following calculation is an element of $\mathfrak{g}$

$$
\begin{aligned}
{\left[A_{x} \oplus_{R, T} x, A_{y} \oplus_{R, T} y\right]=} & \left(\left[A_{x}, A_{y}\right]-R_{x, y}-A_{A_{x} y-A_{y} x-T(x, y)}\right) \oplus_{R, T} 0 \\
& +A_{A_{x} y-A_{y} x-T(x, y)} \oplus_{R, T}\left(A_{x} y-A_{y} x-T(x, y)\right)
\end{aligned}
$$

and thus requires $Q(A, T)_{x, y} \equiv R_{x, y}$ modulo $\mathfrak{h}$ for all $x, y \in V$. In passing we remark that $\left[\mathfrak{X} \oplus_{R, T} 0, \mathfrak{Y} \oplus_{R, T} 0\right]=[\mathfrak{X}, \mathfrak{Y}] \oplus_{R, T} 0$ lies in $\mathfrak{g}$ for $\mathfrak{X}, \mathfrak{Y} \in \mathfrak{h}$ without further ado.

In consequence the subspace $\mathfrak{g}$ of equation (6) is a skew subalgebra of End $V \oplus_{R, T} V$ as soon as $\mathfrak{h} \star A \in V^{*} \otimes \mathfrak{h}$ and $Q(A, T)-R \in \Lambda^{2} V^{*} \otimes \mathfrak{h}$. On the other hand the skew bracket on End $V \oplus_{R, T} V$ agrees with the Lie bracket of the semidirect product End $V \oplus V$ of End $V$ with its representation $V$ up to terms quadratic in $V$. Hence the Jacobiator on every skew subalgebra $\mathfrak{g} \subset$ End $V \oplus_{R, T} V$ vanishes automatically for two or three arguments in the isotropy subalgebra $\mathfrak{h} \subset$ End $V$. In light of this observation the Jacobiator satisfies

$$
\begin{aligned}
\operatorname{Jac}\left(\mathfrak{X} \oplus_{R, T} 0, A_{y} \oplus_{R, T} y, A_{z} \oplus_{R, T} z\right) & =\operatorname{Jac}\left(\mathfrak{X} \oplus_{R, T} 0,0 \oplus_{R, T} y, 0 \oplus_{R, T} z\right) \\
& =-\left((\mathfrak{X} \star R)_{y, z} \oplus_{R, T}(\mathfrak{X} \star T)(y, z)\right)
\end{aligned}
$$


for all $X \in \mathfrak{h}$ and $y, z \in V$, thus the isotropy algebra of a Lie subalgebra $\mathfrak{g} \subset$ End $V \oplus_{R, T} V$ projecting onto $V$ is necessarily a subalgebra $\mathfrak{h} \subset \mathfrak{s t a b} R \cap \mathfrak{s t a b} T$ of the joint stabilizer of $R$ and $T$ in End $V$. Eventually we calculate along a similar line of argument

$$
\begin{aligned}
\operatorname{Jac} & \left(A_{x} \oplus_{R, T} x, A_{y} \oplus_{R, T} y, A_{z} \oplus_{R, T} z\right) \\
= & +\operatorname{Jac}\left(A_{x} \oplus_{R, T} 0,0 \oplus_{R, T} y, 0 \oplus_{R, T} z\right)+\operatorname{Jac}\left(A_{y} \oplus_{R, T} 0,0 \oplus_{R, T} z, 0 \oplus_{R, T} x\right) \\
& +\operatorname{Jac}\left(A_{z} \oplus_{R, T} 0,0 \oplus_{R, T} x, 0 \oplus_{R, T} y\right)+\operatorname{Jac}\left(0 \oplus_{R, T} x, 0 \oplus_{R, T} y, 0 \oplus_{R, T} z\right) \\
= & \left(-d^{(A, T)} R\right)_{x, y, z} \oplus_{R, T}\left(R \wedge \mathrm{id}-d^{(A, T)} T\right)(x, y, z)
\end{aligned}
$$

using trilinearity, cyclic invariance and the explicit formulas calculated above for Jac.

Although the preceeding lemma is reasonably explicit, it is certainly not satisfactory in that we would prefer conditions on the triple $(A, R, T)$ alone, which guarantee the existence of a Lie subalgebra $\mathfrak{g}$ of End $V \oplus_{R, T} V$ satisfying $\mathfrak{g} \supset\left\{A_{x} \oplus_{R, T} x \mid x \in V\right\}$. In order to achieve such a reformulation of Lemma 4.7 let us have another look at the stabilizer filtration

$$
\text { End } V=\ldots=\mathfrak{h}_{-1} \supsetneq \mathfrak{h}_{0} \supsetneq \ldots \supsetneq \mathfrak{h}_{s-1} \supsetneq \mathfrak{h}_{s}=\mathfrak{h}_{s+1}=\ldots=\mathfrak{h}_{\infty} \supseteq \mathfrak{h}
$$

constructed in Definition 4.4. Evidently the connection component $A \in V^{*} \otimes$ End $V$ of the triple $(A, R, T)$ allows us to define for every subalgebra $\hat{\mathfrak{h}} \subset$ End $V$ the derived subalgebra:

$$
\hat{\mathfrak{h}}^{\prime}:=\left\{\mathfrak{X} \in \hat{\mathfrak{h}} \mid\left[\mathfrak{X}, A_{x}\right] \equiv A_{\mathfrak{X} x} \bmod \hat{\mathfrak{h}} \text { for all } x \in V\right\}
$$

For every subalgebra $\hat{\mathfrak{h}} \subset$ End $V$ the quotient End $V / \hat{\mathfrak{h}}$ is naturally a representation of $\hat{\mathfrak{h}}$ and the derived subalgebra $\hat{\mathfrak{h}}^{\prime}$ is nothing else but the stabilizer of the class $A+V^{*} \otimes \hat{\mathfrak{h}}$ represented by $A$ in $V^{*} \otimes($ End $V / \hat{\mathfrak{h}})$. In particular the derived subalgebra is monotone

$$
\hat{\mathfrak{h}}_{\text {small }} \subset \hat{\mathfrak{h}}_{\text {large }} \quad \Longrightarrow \quad \hat{\mathfrak{h}}_{\text {small }}^{\prime} \subset \hat{\mathfrak{h}}_{\text {large }}^{\prime}
$$

because the canonical projection $V^{*} \otimes\left(\right.$ End $\left.V / \hat{\mathfrak{h}}_{\text {small }}\right) \longrightarrow V^{*} \otimes\left(\right.$ End $\left.V / \hat{\mathfrak{h}}_{\text {large }}\right)$ is equivariant under every subalgebra $\hat{\mathfrak{h}}_{\text {small }} \subset \hat{\mathfrak{h}}_{\text {large }}$ so that $\mathfrak{X} \in \hat{\mathfrak{h}}_{\text {small }}$ stabilizing the class represented by $A$ in $V^{*} \otimes\left(\right.$ End $\left.V / \hat{\mathfrak{h}}_{\text {small }}\right)$ still stabilizes its image in $V^{*} \otimes\left(\right.$ End $\left.V / \hat{\mathfrak{h}}_{\text {large }}\right)$.

Thinking of the derived subalgebra construction as a dynamical system $\hat{\mathfrak{h}} \longmapsto \hat{\mathfrak{h}}^{\prime}$ on the set of subalgebras $\hat{\mathfrak{h}} \subset$ End $V$ we observe that Lemma 4.7 is actually asking for the isotropy algebra $\mathfrak{h}=\mathfrak{g} \cap$ End $V$ of a Lie subalgebra $\mathfrak{g} \subset$ End $V \oplus_{R, T} V$ to be a fixed point $\mathfrak{h}=\mathfrak{h}^{\prime}$ contained in $\mathfrak{s t a b} R \cap \mathfrak{s t a b} T$ while at the same time containing all the values of $Q(A, T)-R \in \Lambda^{2} V^{*} \otimes \mathfrak{h}$. The latter condition however becomes ever the more restrictive the smaller is $\mathfrak{h}$, hence the best we can hope for is that the unique maximal fixed point $\mathfrak{h}_{\max }$ of the dynamical system $\hat{\mathfrak{h}} \longmapsto \hat{\mathfrak{h}}^{\prime}$ contained in $\mathfrak{s t a b} R \cap \mathfrak{s t a b} T$ is a sufficiently large subalgebra of End $V$ to satisfy $Q(A, T)-R \in \Lambda^{2} V^{*} \otimes \mathfrak{h}_{\max }$.

The existence of this unique maximal fixed point $\mathfrak{h}_{\max } \subset \mathfrak{s t a b} R \cap \mathfrak{s t a b} T$ is guaranteed simply by the monotonicity of the dynamical system $\hat{\mathfrak{h}} \longmapsto \hat{\mathfrak{h}}^{\prime}$, to wit $\mathfrak{h}_{\max }=\mathfrak{h}_{\infty}$ is necessarily equal to the limit of the filtration sequence (7) starting in $\mathfrak{h}_{0}:=\mathfrak{s t a b} R \cap \mathfrak{s t a b} T$ and 
iterating $\mathfrak{h}_{r+1}:=\mathfrak{h}_{r}^{\prime}$ the derived subalgebra construction for $r \geq 0$. Depending on the curvature-torsion tuple $(R, T)$ this unique maximal fixed point $\mathfrak{h}_{\max }$ may or may not satisfy $Q(A, T)-R \in \Lambda^{2} V^{*} \otimes \mathfrak{h}_{\max }$. Provided the maximal fixed point $\mathfrak{h}_{\max }$ of the dynamical system $\hat{\mathfrak{h}} \longmapsto \hat{\mathfrak{h}}^{\prime}$ contained in stab $R \cap \mathfrak{s t a b} T$ satisfies $Q(A, T)-R \in \Lambda^{2} V^{*} \otimes \mathfrak{h}_{\text {max }}$ there may of course exist other fixed points $\mathfrak{h} \subset \mathfrak{h}_{\max }$ still satisfying $Q(A, T)-R \in \Lambda^{2} V^{*} \otimes \mathfrak{h}$. In geometric terms these additional fixed points correspond to subgroups $G \subset G_{\max }$ still acting transitively on the affine homogeneous space $G_{\max } / H_{\max }$.

In any case the unique maximal fixed point $\mathfrak{h}_{\max }=\mathfrak{h}_{\infty}$ contained in $\mathfrak{s t a b} R \cap \mathfrak{s t a b} T$ agrees with the joint stabilizer of all iterated covariant derivatives $\nabla^{r} T$ and $\nabla^{r} R$ for all $r \geq 0$ together according to Lemma 4.5. In consequence the decisive congruence $Q(A, T)_{x, y} \equiv R_{x, y}$ modulo $\mathfrak{h}_{\max }$ is equivalent to the following set of algebraic equations:

\section{Theorem 4.8 (Algebraic Variety of Affine Homogeneous Spaces)}

For a given connection-curvature-torsion triple $(A, R, T)$ on a vector space $V$ there exists a Lie subalgebra $\mathfrak{g} \subset$ End $V \oplus_{R, T} V$ of the skew algebra associated to $(R, T)$ satisfying

$$
\mathfrak{g} \supset \operatorname{im}\left(A^{\text {ext }}: V \longrightarrow \text { End } V \oplus_{R, T} V, \quad x \longmapsto A_{x} \oplus_{R, T} x\right)
$$

if and only if $(A, R, T)$ satisfies the first and second Bianchi identities of degrees 2 and 3

$$
d^{(A, T)} T=R \wedge \text { id } \quad d^{(A, T)} R=0
$$

as well as the following homogeneous equations of degrees $r+3$ and $r+4$ for all $r \geq 0$ :

$$
\begin{aligned}
& (Q(A, T)-R) \circledast(\underbrace{A \circledast(A \circledast(\ldots(A}_{r \text { times }} \circledast T) \ldots)))=0 \\
& (Q(A, T)-R) \circledast(\underbrace{A \circledast(A \circledast(\ldots(A}_{r \text { times }} \circledast R) \ldots)))=0
\end{aligned}
$$

The set of all solution triples $(A, R, T)$ to these algebraic equations will be denoted $\mathfrak{M}(\mathfrak{g l} V)$.

Although the algebraic variety $\mathfrak{M}(\mathfrak{g l} V)$ is formally defined by an infinite set of algebraic equations, only a finite number of these equations can actually be relevant, after all polynomial rings are noetherian rings. Taking the argument leading to these equations into account we observe that at least the equations parametrized by $r \geq(\operatorname{dim} V)^{2}$ have to be algebraic consequences of the equations up to $(\operatorname{dim} V)^{2}$. In this observation $(\operatorname{dim} V)^{2}$ enters simply as a trivial upper bound for the maximal length $s$ of a sequence $\hat{\mathfrak{h}}_{0} \supsetneq \hat{\mathfrak{h}}_{1} \supsetneq \ldots \supsetneq \hat{\mathfrak{h}}_{s}$ of subalgebras of End $V$ with $\hat{\mathfrak{h}}_{r+1}=\hat{\mathfrak{h}}_{r}^{\prime}$ for some $A \in V^{*} \otimes$ End $V$, which probably grows more like $\operatorname{dim} V$ than $(\operatorname{dim} V)^{2}$. On the other hand the family of examples constructed in Section 6 shows that there is no universal bound independent of $\operatorname{dim} V$ for the number of algebraic equations needed in order to define $\mathfrak{M}(\mathfrak{g l} V)$.

Somewhat more interesting from the theoretical point of view is the observation that the algebraic variety $\mathfrak{M}(\mathfrak{g l} V)$ is actually a cone over a projective algebraic variety due to the 
homogeneity of the algebraic equations defining $\mathfrak{M}(\mathfrak{g l} V)$. More precisely the group $\mathbb{R}$ acts via $\lambda \star(A, R, T):=\left(e^{\lambda} A, e^{2 \lambda} R, e^{\lambda} T\right)$ on the set of all connection-curvature-torsion triples and thus on the invariant subset $\mathfrak{M}(\mathfrak{g l} V) \subset V^{*} \otimes$ End $V \times \Lambda^{2} V^{*} \otimes$ End $V \times \Lambda^{2} V^{*} \otimes V$. The same observation applies to the algebraic varieties $\mathfrak{M}^{\mathrm{tf}}(\mathfrak{g l} V)$ and $\mathfrak{M}^{\mathrm{ref}}(\mathfrak{g l} V)$ of torsion free and reductive connection-curvature-torsion triples $(A, R, T)$ defined in:

\section{Corollary 4.9 (Torsion Free Affine Homogeneous Spaces)}

Torsion free connection-curvature tuples $(A, R)$ are connection-curvature-torsion triples with vanishing torsion $T=0$. Their algebras End $V \oplus_{R, 0} V$ allow Lie subalgebras $\mathfrak{g}$ with

$$
\text { End } V \oplus_{R, 0} V \supset \mathfrak{g} \supset \operatorname{im}\left(A^{\text {ext }}: V \longrightarrow \text { End } V \oplus_{R, 0} V, \quad x \longmapsto A_{x} \oplus_{R, 0} x\right)
$$

if and only if the curvature $R \in \Lambda^{2} V^{*} \otimes$ End $V$ and the connection $A \in V^{*} \otimes$ End $V$ satisfy the formally infinite system of algebraic equations of degrees 2,3 and $r+4, r \geq 0$ :

$\left.\left.\left.R \wedge \mathrm{id}=0 \quad d^{(A, 0)} R=0 \quad(Q(A, 0)-R) \circledast(\underbrace{A \circledast(A \circledast(\ldots(A}_{r \text { times }} \circledast R) \ldots\right)\right)\right)=0$

The set of all solutions $(A, R)$ to these equations will be denoted by $\mathfrak{M}^{\mathrm{tf}}(\mathfrak{g l} V) \subset \mathfrak{M}(\mathfrak{g l} V)$.

\section{Corollary 4.10 (Reductive Affine Homogeneous Spaces)}

Reductive curvature-torsion tuples $(R, T)$ are connection-curvature-torsion triples with vanishing connection $A=0$. The skew algebra End $V \oplus_{R, T} V$ allows Lie subalgebras $\mathfrak{g} \supset V$ extending $V$, if and only if the curvature $R \in \Lambda^{2} V^{*} \otimes$ End $V$ and torsion $T \in \Lambda^{2} V^{*} \otimes V$ satisfy the following system of algebraic equations of degrees 2, 3, 3 and 4:

$$
d^{(0, T)} T=R \wedge \text { id } \quad d^{(0, T)} R=0 \quad R \circledast T=0 \quad R \circledast R=0
$$

In the sequel $\mathfrak{M}^{\mathrm{red}}(\mathfrak{g l} V) \subset \mathfrak{M}(\mathfrak{g l} V)$ will denote the set of solutions to these equations.

\section{$5 \quad$ Additional Parallel Geometric Structures}

In this short intermediate section we want to discuss variations of the algebraic varieties $\mathfrak{M}(\mathfrak{g l} V)$ parametrizing formal affine homogeneous spaces, which take into account additional parallel geometric structures like Riemannian metrics and almost complex structures. Because the affine geometry determined by the existence of a linear connection of the tangent bundle is intrisically a first order geometry, the condition of parallelity severely restricts the order of geometric structures we may consider in addition, in any case we will restrict ourselves to a discussion of first order geometries usually called (first order) $G$-structures. For the purpose of this article we prefer to call them $K$-structures instead in order to avoid the clash of notation with the big group $G$ of the homogeneous space $G / H$.

In order to define $K$-structures on vector spaces and consequently on smooth manifolds $M$ we fix once and for all a closed subgroup $K \subset$ GL $V$ of the general linear group of a vector 
space $V$ called the model space. By definition a $K$-structure on a vector space $T$ of the same dimension as $V$ is an equivalence class $\Omega$ of linear isomorphisms $F: V \longrightarrow T$ under

$$
F \sim_{K} \tilde{F} \Longleftrightarrow F^{-1} \circ \tilde{F} \in K
$$

conversely the linear isomorphisms $F: V \longrightarrow T$ representing $\Omega$ are called $K$-frames. The general linear group GL $V$ acts simply transitively from the right on the set Frame $(V, T)$ of linear isomorphisms $V \longrightarrow T$, thus $K$-structures $\Omega$ correspond bijectively to points in:

$$
\Omega \in \operatorname{Frame}(V, T) / K
$$

With $K$ being a closed subgroup of GL $V$ by assumption the quotient $\operatorname{Frame}(V, T) / K$ is actually a manifold so that we can define a smooth $K$-structure on a manifold $M$ of the same dimension as $V$ as a smooth section of the quotient bundle

$$
\Omega \in \Gamma(\operatorname{Frame}(V, T M) / K)
$$

where Frame $(V, T M)$ is the standard principal GL $V$-bundle of all frames on $M$. Such a smooth $K$-structure $\Omega$ is parallel for an affine connection $\nabla$ on $T M$, if and only if the parallel transport $\mathrm{PT}_{\gamma}^{\nabla}: T_{\gamma(0)} M \longrightarrow T_{\gamma(1)} M$ along arbitrary curves $\gamma:[0,1] \longrightarrow M$ maps $K$-frames to $K$-frames in the sense $\mathrm{PT}_{\gamma}^{\nabla} \circ F \in \Omega_{\gamma(1)}$ for every $K$-frame $F \in \Omega_{\gamma(0)}$.

Whereas the preceeding definition of parallel $K$-structures on a manifold $M$ for a closed subgroup $K \subset$ GL $V$ does not comprise the most general geometric structures, nevertheless there are quite a number of interesting examples. Consider for example $V=\mathbb{C}^{n}$ as a real vector space of dimension $2 n$, which inherits from $\mathbb{C}^{n}$ the complex structure $I \in$ End $V$, the real part $g \in \operatorname{Sym}_{+}^{2} V^{*}$ of the standard hermitean form $(\cdot, \cdot)$ and the $n$-form $\psi \in \Lambda^{n} V^{*}$ :

$$
\psi\left(v_{1}, \ldots, v_{n}\right):=\operatorname{Re} \operatorname{det}_{\mathbb{C}}\left(v_{1}, \ldots, v_{n}\right)
$$

The common stabilizer of the triple $(g, I, \psi)$ agrees with the subgroup $\mathbf{S U}(n) \subset$ GL $V$ so that an $\mathbf{S U}(n)$-structure $\Omega$ on a $2 n$-dimensional vector space defines a corresponding triple

$$
g_{\Omega}:=g\left(F^{-1} \cdot, F^{-1} \cdot\right) \quad I_{\Omega}:=F \circ I \circ F^{-1} \quad \psi_{\Omega}:=\psi\left(F^{-1} \cdot \ldots, F^{-1} \cdot\right)
$$

on $T$ for any representative $F \in \Omega$. On the other hand the model triple $(g, I, \psi)$ satisfies

$$
I^{2}=-\operatorname{id}_{V} \quad g(I \cdot, I \cdot)=g \quad \operatorname{Der}_{I}^{2} \psi=-n^{2} \psi \quad g^{-1}(\psi, \psi)=2^{n-1}
$$

and it can be shown straightforwardly that GL $V$ acts transitively on the set of solutions to these algebraic equations in $\operatorname{Sym}_{+}^{2} V^{*} \times$ End $V \times \Lambda^{n} V^{*}$. In consequence $\mathbf{S U}(n)$-structures $\Omega$ on a vector space $T$ are in bijection to triples $(g, I, \psi)$ in $\operatorname{Sym}_{+}^{2} T^{*} \times \operatorname{End} T \times \Lambda^{n} T^{*}$ satisfying the algebraic equations (8). Similarly the tuple $(g, I)$ defines the underlying $\mathbf{U}(n)$-structure and $g \in \operatorname{Sym}_{+}^{2} T^{*}$ only the underlying $\mathbf{O}(2 n)$-structure on $T$.

Coming back to homogeneous spaces $G / H$ we define a left invariant $K$-structure as a left invariant section of the homogeneous quotient bundle Frame $(V, T(G / H)) / K$ of the homogeneous frame bundle. By the classification of left invariant sections such a section $\Omega$ is completely determined by its $H$-invariant value in the base point $e H \in G / H$ of $G / H$

$$
\Omega_{e H} \in[\operatorname{Frame}(V, \mathfrak{g} / \mathfrak{h}) / K]^{H}
$$


where the condition of $H$-invariance reads for a representative $F: V \longrightarrow \mathfrak{g} / \mathfrak{h}$ of $\Omega_{e H}$

$$
(h \star) \circ F \sim_{K} F \quad \Longleftrightarrow \quad F^{-1} \circ(h \star) \circ F \in K
$$

for every $h \in H$, equivalently the image $\operatorname{Ad} H \subset \mathbf{G L} \mathfrak{g} / \mathfrak{h}$ of $H$ under the adjoint representation must be conjugated under $F$ to the subgroup $F^{-1}(\mathrm{Ad} H) F \subset K$ of $K$. Similarly a left invariant $K$-structure on $G / H$ is parallel for a left invariant connection $A: \mathfrak{g} \longrightarrow$ End $\mathfrak{g} / \mathfrak{h}$ on $T(G / H)$ provided $F^{-1} \circ A_{X} \circ F$ is an element of the Lie algebra $\mathfrak{k}$ of $K$ for every $X \in \mathfrak{g}$.

In consequence we may define a formal affine homogeneous space with parallel $K-$ structure as a pair of Lie algebras $\mathfrak{g} \supset \mathfrak{h}$ endowed with a formal left in variant connection $A: \mathfrak{g} \longrightarrow$ End $\mathfrak{g} / \mathfrak{h}$ and a $K$-structure $\Omega$ on $\mathfrak{g} / \mathfrak{h}$ such that $F^{-1} \circ(H \star) \circ F \subset \mathfrak{k}$ and $F^{-1} \circ A_{X} \circ F \in \mathfrak{k}$ for all $H \in \mathfrak{h}$ and $X \in \mathfrak{g}$. On the other hand we recall that we need to choose a frame $F: V \longrightarrow \mathfrak{g} / \mathfrak{h}$ anyhow in order to associate to such a formal homogeneous space a point in the algebraic variety $\mathfrak{M}(\mathfrak{g l} V)$, making the straightforward choice of a frame $F$ representing $\Omega$ we arrive at the algebraic variety of formal affine homogeneous spaces

$$
\mathfrak{M}(\mathfrak{k}):=\mathfrak{M}(\mathfrak{g l} V) \cap\left(V^{*} \otimes \mathfrak{k} \times \Lambda^{2} V^{*} \otimes \mathfrak{k} \times \Lambda^{2} V^{*} \otimes V\right)
$$

with parallel $K$-structure. Passing through the arguments used in the construction of the algebraic variety $\mathfrak{M}(\mathfrak{g l} V)$ we conclude that the connection-curvature-torsion triples $(A, R, T)$ in $\mathfrak{M}(\mathfrak{k})$ correspond to maximal Lie subalgebras $\mathfrak{g} \subset \mathfrak{k} \oplus_{R, T} V$ of the skew algebras $\mathfrak{k} \oplus_{R, T} V$, which contain the image of the extended connection $V \longrightarrow \mathfrak{k} \oplus_{R, T} V, x \longmapsto A_{x} \oplus_{R, T} x$. Similar considerations apply of course to the algebraic varieties $\mathfrak{M}^{\mathrm{tf}}(\mathfrak{k})$ and $\mathfrak{M}^{\text {red }}(\mathfrak{k})$ of torsion free and reductive formal affine homogeneous spaces with parallel $K$-structures.

\section{Contact Order and Formal Tangent Space}

In a sense the algebraic variety $\mathfrak{M}(\mathfrak{g l} V)$ of affine homogeneous spaces and its variants are only coarse moduli spaces, because they parametrize isometry classes of formal affine homogeneous spaces $\mathfrak{g} \supset \mathfrak{h}$ augmented by a frame isomorphism $F: V \longrightarrow \mathfrak{g} / \mathfrak{h}$ and a split $\mathfrak{g} / \mathfrak{h} \longrightarrow \mathfrak{g}$ of the canonical projection. The effect of changing the frame and/or the split introduces an equivalence relation on $\mathfrak{M}(\mathfrak{g l} V)$, the equivalence relation $\sim_{\infty}$ of contact to all orders, which is approximated by the equivalence relations $\sim_{d}$ of contact to order $d \in \mathbb{N}_{0}$, In turn the formal tangent space to the true moduli space $\mathfrak{M}_{\infty}(\mathfrak{g l} V):=\mathfrak{M}(\mathfrak{g l} V) / \sim_{\infty}$ in a point $[A, R, T]$ is filtered by directions staying in contact with $[A, R, T]$ up to order $d \geq 0$.

After introducing the contact equivalence relations $\sim_{d}$ and $\sim_{\infty}$ we use the stabilizer filtration of Definition 4.4 in order to associate to every connection-curvature-torsion triple $(A, R, T) \in \mathfrak{M}(\mathfrak{g l} V)$ its Spencer cohomology $H^{\bullet, \circ}(\mathfrak{h})$. Moreover we identify this Spencer cohomology with the sucessive filtration quotients of the contact filtration on the formal tangent space $T_{[A, R, T]} \mathfrak{M}_{\infty}(\mathfrak{g l} V)$ to the true moduli space $\mathfrak{M}_{\infty}(\mathfrak{g l} V)$ in the point $[A, R, T]$. Eventually we will illustrate this interpretation of the Spencer cohomology associated to $(A, R, T)$ by detailed calculations for the family of pairwise non-isometric Riemannian homogeneous spaces with large Singer invariant constructed by Meusers [M]. 


\section{Definition 6.1 (Contact Relation for Connection-Curvature-Torsion Triples)}

Two connection-curvature-torsion triples $(A, R, T)$ and $(\tilde{A}, \tilde{R}, \tilde{T})$ on a finite-dimensional vector space $V$ are said to be in contact to order $d \geq 0$ written $(A, R, T) \sim_{d}(\tilde{A}, \tilde{R}, \tilde{T})$, if there exists a linear automorphism $F \in \mathbf{G L} V$ of $V$ pulling the formal covariant derivatives of $\tilde{R}$ and $\tilde{T}$ of all orders $r=0, \ldots, d$ back to the formal covariant derivatives of $R$ and $T$ :

$$
\begin{aligned}
& \left.\left.F^{*}(\underbrace{\tilde{A} \circledast(\tilde{A} \circledast \ldots(\tilde{A}}_{r \text { times }} \circledast \tilde{T}) \ldots\right)\right)=\underbrace{A \circledast(A \circledast \ldots(A}_{r \text { times }} \circledast T) \ldots) \\
& \left.\left.F^{*}(\underbrace{\tilde{A} \circledast(\tilde{A} \circledast \ldots(\tilde{A}}_{r \text { times }} \circledast \tilde{R}) \ldots\right)\right)=\underbrace{A \circledast(A \circledast \ldots(A}_{r \text { times }} \circledast R) \ldots)
\end{aligned}
$$

Similar the notation $(A, R, T) \sim_{\infty}(\tilde{A}, \tilde{R}, \tilde{T})$ indicates triples in contact to all orders $d \geq 0$.

The naturality of the operation $\circledast$ implies of course for the iterated covariant derivatives

$$
F^{*}(A \circledast(A \circledast \ldots(A \circledast T) \ldots))=F^{*} A \circledast\left(F^{*} A \circledast \ldots\left(F^{*} A \circledast F^{*} T\right) \ldots\right)
$$

of $T$ or similarly of $R$. The main argument of Lemma 4.5 may thus be varied to prove:

\section{Lemma 6.2 (Explicit Form of the Contact Relation)}

Two connection-curvature-torsion triples $(A, R, T)$ and $(\tilde{A}, \tilde{R}, \tilde{T})$ on a finite-dimensional vector space $V$ are in contact $(A, R, T) \sim_{d}(\tilde{A}, \tilde{R}, \tilde{T})$ to order $d \geq 0$, if and only if there exists a linear automorphism $F \in \mathbf{G L} V$ satisfying $F^{*} \tilde{T}=T$ and $F^{*} \tilde{R}=R$ as well as:

$$
F^{*} \tilde{A} \equiv A \quad \bmod V^{*} \otimes \mathfrak{h}_{d-1}
$$

In consequence two triples $(A, R, T) \sim_{d}(\tilde{A}, \tilde{R}, \tilde{T})$ in contact to an order $d>\operatorname{Singer}(A, R, T)$ exceeding the Singer invariant of one are in contact $(A, R, T) \sim_{\infty}(\tilde{A}, \tilde{R}, \tilde{T})$ to all orders.

The infinite order contact relation $\sim_{\infty}$ reflects exactly the dependence of the connectioncurvature-torsion triple $(A, R, T)$ associated to a formal affine homogeneous space $\mathfrak{g} \supset \mathfrak{h}$ on the additional choice of frame $F: V \longrightarrow \mathfrak{g} / \mathfrak{h}$ and split $\mathfrak{g} / \mathfrak{h} \longrightarrow \mathfrak{g}$ of the canonical projection. The moduli space of isometry classes of formal affine homogeneous spaces may thus be defined as the set of equivalence classes of points $(A, R, T) \in \mathfrak{M}(\mathfrak{g l} V)$ under $\sim_{\infty}$ :

$$
\mathfrak{M}_{\infty}(\mathfrak{g l} V):=\mathfrak{M}(\mathfrak{g l} V) / \sim_{\infty}
$$

Similar definitions can be made for the moduli spaces of torsion free $\mathfrak{M}_{\infty}^{\mathrm{tf}}(\mathfrak{g l} V)$ or reductive formal affine homogeneous spaces $\mathfrak{M}_{\infty}^{\text {red }}(\mathfrak{g l} V)$ with or without additional left invariant parallel $K$-structures. It turns out that the deformation theory of isometry classes of formal affine homogeneous spaces considered as points in $\mathfrak{M}_{\infty}(\mathfrak{g l} V)$ is governed by a suitable version of the Spencer cohomology associated to the purely algebraic concept of a graded comodule over the symmetric coalgebra Sym $V^{*}$ of a vector space $V$ : 


\section{Definition 6.3 (Comodules over Symmetric Coalgebras)}

A comodul over the symmetric coalgebra Sym $V^{*}$ of a finite-dimensional vector space $V$ is a $\mathbb{Z}$-graded vector space $\mathscr{A} \bullet$ together with a bilinear map called directional derivative $V \times \mathscr{A}^{\bullet} \longrightarrow \mathscr{A}^{\bullet-1},(y, \mathfrak{X}) \longmapsto \frac{\partial \mathfrak{X}}{\partial y}$, homogeneous of degree -1 with respect to $\mathscr{A}$ such that

$$
\frac{\partial}{\partial y}\left(\frac{\partial \mathfrak{X}}{\partial z}\right)=\frac{\partial}{\partial z}\left(\frac{\partial \mathfrak{X}}{\partial y}\right)
$$

for all $y, z \in V$. In particular the iterated directional derivatives like $\frac{\partial^{2} \mathfrak{X}}{\partial y \partial z}$ are well defined.

\section{Definition 6.4 (Spencer Complex and Cohomology)}

The Spencer complex associated to a comodule $\mathscr{A}$ over the symmetric coalgebra Sym $V^{*}$ of a finite-dimensional vector space $V$ is the bigraded complex of alternating multilinear forms $\Lambda^{\circ} V^{*} \otimes \mathscr{A}^{\bullet}$ on $V$ with values in the comodule $\mathscr{A}$

$$
\ldots \stackrel{B}{\longrightarrow} \Lambda^{\circ-1} V^{*} \otimes \mathscr{A}^{\bullet+1} \stackrel{B}{\longrightarrow} \Lambda^{\circ} V^{*} \otimes \mathscr{A}^{\bullet} \stackrel{B}{\longrightarrow} \Lambda^{\circ+1} V^{*} \otimes \mathscr{A}^{\bullet-1} \stackrel{B}{\longrightarrow} \ldots
$$

endowed with the Spencer coboundary operator B defined for $\eta \in \Lambda^{k} V^{*} \otimes \mathscr{A}^{\bullet}$ by:

$$
(B \eta)\left(x_{0}, \ldots, x_{k}\right):=\sum_{\mu=0}^{k}(-1)^{\mu} \frac{\partial}{\partial x_{\mu}} B\left(x_{0}, \ldots, \widehat{x_{\mu}}, \ldots, x_{k}\right)
$$

The corresponding bigraded cohomology theory for comodules is called Spencer cohomology:

$$
H^{\bullet, \circ}(\mathscr{A}):=\frac{\operatorname{ker}\left(B: \mathscr{A}^{\bullet} \otimes \Lambda^{\circ} V^{*} \longrightarrow \mathscr{A}^{\bullet-1} \otimes \Lambda^{\circ+1} V^{*}\right)}{\operatorname{im}\left(B: \mathscr{A}^{\bullet+1} \otimes \Lambda^{\circ-1} V^{*} \longrightarrow \mathscr{A}^{\bullet} \otimes \Lambda^{\circ} V^{*}\right)}
$$

A detailed description of the general properties of the Spencer cohomology of comodules is certainly beyond the scope of this article, for more information see for example [BCG], [W]. Nevertheless we want to point out that the Spencer cohomology $H^{\bullet \bullet \circ}(\mathscr{A})$ of a comodule $\mathscr{A}^{\bullet}$ is naturally a graded right module over the exterior algebra $\Lambda^{\circ} V^{*}$. For a comodule $\mathscr{A}$ constant in the directions of a subspace $W \subset V$ in the sense $\frac{\partial \mathfrak{X}}{\partial x}=0$ for all $\mathfrak{X} \in \mathscr{A}$ and all $x \in W$ for example the right $\Lambda^{\circ} V^{*}$-module structure turns out to be convenient to prove

$$
H^{\bullet, \circ}(\mathscr{A}) \cong H_{V / W}^{\bullet \circ}(\mathscr{A}) \otimes \Lambda^{\circ} W^{*}
$$

where $H_{V / W}^{\bullet}, \circ(\mathscr{A})$ refers to the Spencer cohomology of $\mathscr{A}^{\bullet}$ considered as a comodule over the coalgebra Sym $(V / W)^{*}$ and the repeated grading symbol $\circ$ indicates the product grading.

\section{Definition 6.5 (Formal Directional Derivatives)}

Consider the strictly decreasing filtration (7) associated to a connection-curvature-torsion triple $(A, R, T)$ in the variety $\mathfrak{M}(\mathfrak{g l} V)$. The direct sum of sucessive filtration quotients

$$
\mathfrak{h}^{\bullet}=\bigoplus_{r \in \mathbb{Z}}\left(\mathfrak{h}_{r-1} / \mathfrak{h}_{r}\right)=\left(\text { End } V / \mathfrak{h}_{0}\right) \oplus \ldots \oplus\left(\mathfrak{h}_{s-1} / \mathfrak{h}_{\infty}\right)
$$


is a comodule over the symmetric coalgebra Sym $V^{*}$ under directional derivatives defined by

$$
\frac{\partial \mathfrak{X}}{\partial x}:=\left[\mathfrak{X}, A_{x}\right]-A_{\mathfrak{X} x}+\mathfrak{h}_{r-1}
$$

for $x \in V$ and all representatives $\mathfrak{X} \in \mathfrak{h}_{r-1}$ of a class $\mathfrak{X}+\mathfrak{h}_{r}$ in the quotient $\mathfrak{h}^{r}:=\mathfrak{h}_{r-1} / \mathfrak{h}_{r}$.

Of course we should not forget to verify the axiomatic commutation of the formal directional derivatives for a comodule over the symmetric coalgebra Sym $V^{*}$, which is quite surprising in view of the complicated definition of the comodule $\mathfrak{h}^{\bullet}$ and its formal directional derivatives. Straightforward calculation of iterated formal derivatives results in the not too pleasant

$$
\begin{aligned}
\frac{\partial}{\partial y} \frac{\partial \mathfrak{X}}{\partial z} & \equiv\left[\left[\mathfrak{X}, A_{z}\right]-A_{\mathfrak{X} z}, A_{y}\right]-A_{\left(\left[\mathfrak{x}, A_{z}\right]-A_{\mathfrak{X} z}\right) y} \\
& \equiv-\left[A_{y},\left[\mathfrak{X}, A_{z}\right]\right]+\left[A_{y}, A_{\mathfrak{X} z}\right]-A_{\mathfrak{X}\left(A_{z} y\right)}+A_{A_{z}(\mathfrak{X} y)}+A_{A_{\mathfrak{x} z} y}
\end{aligned}
$$

modulo $\mathfrak{h}_{r-2}$ for a given representative $\mathfrak{X} \in \mathfrak{h}_{r-1}$ of a class $\mathfrak{X}+\mathfrak{h}_{r} \in \mathfrak{h}_{r-1} / \mathfrak{h}_{r}$, in consequence

$$
\begin{aligned}
\frac{\partial}{\partial z} \frac{\partial \mathfrak{X}}{\partial y}-\frac{\partial}{\partial y} \frac{\partial \mathfrak{X}}{\partial z} \equiv & +\left[\mathfrak{X},\left[A_{y}, A_{z}\right]-A_{A_{y} z}+A_{A_{z} y}+A_{T(y, z)}\right]-A_{\mathfrak{X} T(y, z)} \\
& -\left[A_{\mathfrak{X} y}, A_{z}\right]+A_{A_{\mathfrak{X} y} z}-A_{A_{z}(\mathfrak{X} y)}-A_{T(\mathfrak{X} y, z)}+A_{T(\mathfrak{X} y, z)} \\
& -\left[A_{y}, A_{\mathfrak{X} z}\right]+A_{A_{y}(\mathfrak{x} z)}-A_{A_{\mathfrak{x} z} y}-A_{T(y, \mathfrak{X} z)}+A_{T(y, \mathfrak{X} z)} \\
& +\left[\mathfrak{X}, A_{A_{y} z}-A_{A_{z} y}-A_{T(y, z)}\right]-A_{\mathfrak{X}\left(A_{y} z-A_{z} y-T(y, z)\right)} \\
\equiv & {[\mathfrak{X} \star Q(A, T)]_{y . z}+\frac{\partial \mathfrak{X}}{\partial\left(A_{y} z-A_{z} y-T(y, z)\right)}-A_{[\mathfrak{X} \star T](y, z)} }
\end{aligned}
$$

modulo $\mathfrak{h}_{r-2}$. For $r \leq 1$ there is actually nothing to prove, because the right hand side vanishes for trivial reasons due to $\mathfrak{h}_{-1}=$ End $V$. On the other hand $\mathfrak{X} \in \mathfrak{h}_{r-1} \subset \mathfrak{h}_{0}$ for $r \geq 2$ so that $\mathfrak{X} \star T=0$ by the very definition of $\mathfrak{h}_{0}=\mathfrak{s t a b} R \cap \mathfrak{s t a b} T$. The directional derivative term on the right hand side vanishes by definition modulo $\mathfrak{h}_{r-2}$ leaving us with

$$
\frac{\partial}{\partial z} \frac{\partial \mathfrak{X}}{\partial y}-\frac{\partial}{\partial y} \frac{\partial \mathfrak{X}}{\partial z} \equiv[\mathfrak{X} \star(Q(A, T)-R)]_{y, z} \quad \bmod \mathfrak{h}_{r-2}
$$

in view of $\mathfrak{X} \in \mathfrak{s t a b} R$. For a connection-curvature-torsion triple $(A, R, T) \in \mathfrak{M}(\mathfrak{g l} V)$ however the difference $Q(A, T)-R$ is an $\mathfrak{h}_{\infty}$-valued 2 -form on $V$ so that $\mathfrak{X} \star(Q(A, T)-R)$ is actually $\mathfrak{h}_{r-1}$-valued for all $\mathfrak{X} \in \mathfrak{h}_{r-1} \supset \mathfrak{h}_{\infty}$. In consequence the formal directional derivatives of the comodule $\mathfrak{h}^{\bullet}$ defined above do in fact commute and $\mathfrak{h}^{\bullet}$ is a comodule over the symmetric coalgebra Sym $V^{*}$. Its Spencer cohomology in form degree 0 is very simple:

\section{Remark 6.6 (Stabilizer Filtration and Spencer Cohomology)}

The only non-vanishing Spencer cohomology in form degree 0 of the comodule $\mathfrak{h}^{\bullet}$ associated to a connection-curvature-torsion triple $(A, R, T)$ in the algebraic variety $\mathfrak{M}(\mathfrak{g l} V)$ is:

$$
H^{0,0}(\mathfrak{h})=\mathfrak{h}^{0}:=\text { End } V / \mathfrak{h}_{0}
$$

In fact $H^{r, 0}(\mathfrak{h})=\{0\}$ for all positive $r>0$ by the definition the stabilizer filtration (7). 
Whereas the Spencer cohomology in form degree 0 is thus not particularly interesting, the Spencer cohomology $H^{\bullet}, 1(\mathfrak{h})$ in form degree 1 has the following neat geometric interpretation in terms of deformations of a formal affine homogeneous space $\mathfrak{g} \supset \mathfrak{h}$.

\section{Lemma 6.7 (Formal Tangent Space of the Moduli Space)}

Given a point $[A, R, T]$ in the moduli space $\mathfrak{M}_{\infty}(V)$ of formal affine homogeneous spaces of dimension $\operatorname{dim} V$ we may consider the subsets of formal affine homogeneous spaces

$$
\mathfrak{M}_{\infty}^{d}(A, R, T):=\left\{[\tilde{A}, \tilde{R}, \tilde{T}] \mid[\tilde{A}, \tilde{R}, \tilde{T}] \sim_{d}[A, R, T]\right\} \subset \mathfrak{M}_{\infty}(\mathfrak{g l} V)
$$

in contact to $[A, R, T]$ to order $d \geq 0$. The resulting decreasing filtration of $\mathfrak{M}_{\infty}(\mathfrak{g l} V)$

$$
\mathfrak{M}_{\infty}(\mathfrak{g l} V) \supseteq \mathfrak{M}_{\infty}^{0}(A, R, T) \supseteq \mathfrak{M}_{\infty}^{1}(A, R, T) \supseteq \ldots \supseteq \mathfrak{M}_{\infty}^{s}(A, R, T) \supseteq\{[A, R, T]\}
$$

provides an interpretation of the Spencer cohomology of $\mathfrak{h}$ by means of formal tangent spaces:

$$
H^{\bullet}, 1(\mathfrak{h})=T_{[A, R, T]} \mathfrak{M}_{\infty}^{\bullet}(A, R, T) / T_{[A, R, T]} \mathfrak{M}_{\infty}^{\bullet-1}(A, R, T)
$$

Proof: Consider a curve of connection-curvature-torsion triples $\varepsilon \longmapsto\left(A_{\varepsilon}, R_{\varepsilon}, T_{\varepsilon}\right)$ representing a tangent vector to $\mathfrak{M}_{\infty}^{d}(A, R, T)$ in the point $[A, R, T]=\left[A_{0}, R_{0}, T_{0}\right]$ :

$$
\left.\frac{d}{d \varepsilon}\right|_{0}\left[A_{\varepsilon}, R_{\varepsilon}, T_{\varepsilon}\right] \in T_{[A, R, T]} \mathfrak{M}_{\infty}^{d}(A, R, T)
$$

By assumption all triples $\left[A_{\varepsilon}, R_{\varepsilon}, T_{\varepsilon}\right]$ are in contact to order $d \geq 0$ so that we may find a smooth curve $\varepsilon \longmapsto F_{\varepsilon}$ in GL $V$ satisfying $F_{\varepsilon}^{*} T_{\varepsilon}=T$ and $F_{\varepsilon}^{*} R_{\varepsilon}=R$ as well as $F_{\varepsilon}^{*} A_{\varepsilon} \equiv A$ modulo $V^{*} \otimes \mathfrak{h}_{d-1}$. The infinitesimal variation of the curve $\varepsilon \longmapsto\left[A_{\varepsilon}, R_{\varepsilon}, T_{\varepsilon}\right]$ defined by

$$
\delta A:=\left.\frac{d}{d \varepsilon}\right|_{0} F_{\varepsilon}^{*} A_{\varepsilon} \in V^{*} \otimes \mathfrak{h}_{d-1}
$$

clearly satisfies $\delta A \in V^{*} \otimes \mathfrak{h}_{d}$, if the original curve $\varepsilon \longmapsto\left(A_{\varepsilon}, R_{\varepsilon}, T_{\varepsilon}\right)$ stays actually in contact to $[A, R, T]$ to order $d+1$. For this reason we are interested in the class represented by $\delta A$ in $V^{*} \otimes \mathfrak{h}^{d}=V^{*} \otimes\left(\mathfrak{h}_{d-1} / \mathfrak{h}_{d}\right)$. This infinitesimal variation class is closed, because

$$
\begin{aligned}
B(\delta A)(y, z) & \equiv \frac{\partial}{\partial y}\left(\delta A_{z}\right)-\frac{\partial}{\partial z}\left(\delta A_{y}\right) \equiv\left[\delta A_{z}, A_{y}\right]-\left[\delta A_{y}, A_{z}\right]+A_{\delta A_{y} z-\delta A_{z} y} \\
& \equiv-\delta Q(A, T)_{y, z}-\delta A_{A_{y} z-A_{z} y}
\end{aligned}
$$

vanishes modulo $\mathfrak{h}_{d-1}$, where $\delta Q$ is the infinitesimal variation of the approximate curvature:

$$
\delta Q:=\left.\frac{d}{d \varepsilon}\right|_{0} Q\left(F_{\varepsilon}^{*} A_{\varepsilon}, T\right) \in \Lambda^{2} V^{*} \otimes \mathfrak{h}_{d-1}
$$

In fact all connection-curvature-torsion triples $\left(F_{\varepsilon}^{*} A_{\varepsilon}, R, T\right) \in \mathfrak{M}(\mathfrak{g l} V)$ are in contact to order $d \geq 0$ by assumption and thus share the initial part $\mathfrak{h}_{-1} \supsetneq \ldots \supsetneq \mathfrak{h}_{d-1}$ of their stabilizer filtrations by Lemma 4.5. Among the equations defining $\mathfrak{M}(\mathfrak{g l} V)$ is the congruence

$$
Q\left(F_{\varepsilon}^{*} A_{\varepsilon}, T\right) \equiv R \bmod \Lambda^{2} V^{*} \otimes\left(\mathfrak{h}_{\varepsilon}\right)_{\infty}
$$


which implies $Q\left(F_{\varepsilon}^{*} A_{\varepsilon}, T\right) \equiv R$ modulo $\Lambda^{2} V^{*} \otimes \mathfrak{h}_{d-1}$ for all $\varepsilon$ and thus proves the implicit claim in definition (11). Being closed for the Spencer coboundary operator $B$ the infinitesimal variation represents a class $[\delta A] \in H^{d, 1}(\mathfrak{h})$ in the Spencer cohomology of the comodule $\mathfrak{h}^{\bullet}$ associated to $[A, R, T]$. In case this class vanishes and the infinitesimal variation is exact

$$
\delta A=B \mathfrak{X} \equiv \mathfrak{X} \star A \bmod V^{*} \otimes \mathfrak{h}_{d}
$$

for some $\mathfrak{X} \in \mathfrak{h}_{d}$, hence the two curves $\varepsilon \longmapsto\left(e^{-\varepsilon \mathfrak{X}} \star F_{\varepsilon}^{*} A_{\varepsilon}, R, T\right)$ and $\varepsilon \longmapsto\left(A_{\varepsilon}, R_{\varepsilon}, T_{\varepsilon}\right)$ represent the same tangent vector in $[A, R, T]$ tangent to $\mathfrak{M}_{\infty}^{d+1}(\mathfrak{g l} V)$.

In order to illustrate the relation between the Spencer cohomology of Definition 6.4 and the formal tangent spaces to the moduli space $\mathfrak{M}_{\infty}(\mathfrak{g l} V)$ we want to study the family of examples of Riemannian homogeneous spaces with large Singer invariant constructed by Meusers [M] in detail. Calculations can be streamlined significantly using the standard identification of the Lie algebra $\mathfrak{s o} V$ of skew symmetric endomorphisms on a euclidian vector space $V$ with scalar product $g$ with the second exterior power $\Lambda^{2} V$

$$
\Lambda^{2} V \stackrel{\cong}{\longrightarrow} \mathfrak{s o} V, \quad X \wedge Y \longmapsto(Z \longmapsto g(X, Z) Y-g(Y, Z) X)
$$

characterized by $g(\mathfrak{X}, Y \wedge Z)=g(\mathfrak{X} Y, Z)$ for all $Y, Z \in V$ and $\mathfrak{X} \in \Lambda^{2} V$ on the left, but $\mathfrak{X} \in \mathfrak{s o} V$ on the right hand side. The Lie bracket on $\Lambda^{2} V=\mathfrak{s o} V$ satisfies the formulas

$$
[X \wedge Y, X \wedge Z]=g(X, X) Y \wedge Z \quad[\mathfrak{X}, Y \wedge Z]=\mathfrak{X} Y \wedge Z+Y \wedge \mathfrak{X} Z
$$

for all $\mathfrak{X} \in \mathfrak{s o} V$ and all $Y, Z \in V$ satisfying $g(X, Y)=0=g(X, Z)$. Consider now a euclidian vector space $V_{\circ}$ endowed with a scalar product $g \in \operatorname{Sym}_{+}^{2} V^{*}$ and an endomorphism $F: V_{\circ} \longrightarrow V_{\circ}$. The direct sum $V:=\mathbb{R} \oplus V_{\circ}$ is then a euclidian Lie algebra under the extension $g(x \oplus X, y \oplus Y)=x y+g(X, Y)$ of the scalar product and the Lie bracket:

$$
[x \oplus X, y \oplus Y]:=0 \oplus(x F Y-y F X)
$$

Evidently $V$ is a solvable Lie algebra with abelian nilpotent subalgebra $[V, V]=V_{\circ}$, which can be realized as the Lie algebra of left invariant vector fields on the corresponding simply connected solvable Lie group $G$. After a short calculation it turns out that the Levi-Civita connection of the left invariant metric $g$ on $G$ depends on the decomposition of $F=F_{+}+F_{-}$ into its symmetric part $F_{+}$and its skew symmetric part $F_{-}$, more precisely we obtain:

$$
A: \quad V \longrightarrow \text { End } V, \quad x \oplus X \longrightarrow\left(F_{+} X\right) \wedge \mathbf{1}+x F_{-}
$$

In fact $A_{x \oplus X} \in \mathfrak{s o} V$ is a skew symmetric endomorphism of $V$ for all $x \oplus X \in V$ and

$$
\begin{aligned}
& A_{x \oplus X}(y \oplus Y)-A_{y \oplus Y}(x \oplus X) \\
& =\left(g\left(F_{+} X, Y\right)-g\left(F_{+} Y, X\right)\right) \oplus\left(\left(-y F_{+} X+x F_{-} Y\right)-\left(-x F_{+} Y+y F_{-} X\right)\right) \\
& =0 \oplus(x F Y-y F X)=[x \oplus X, y \oplus Y]
\end{aligned}
$$


agrees with the Lie bracket. Using this piece of information we calculate the curvature to be

$$
\begin{aligned}
& R_{x \oplus X, y \oplus Y} \\
& =\left[\left(F_{+} X\right) \wedge \mathbf{1}+x F_{-},\left(F_{+} Y\right) \wedge \mathbf{1}+y F_{-}\right]-\left(x F_{+} F Y-y F_{+} F X\right) \wedge \mathbf{1} \\
& =\left(F_{+} X\right) \wedge\left(F_{+} Y\right)-x\left(F_{+}^{2}+\left[F_{+}, F_{-}\right]\right) Y \wedge \mathbf{1}+y\left(F_{+}^{2}+\left[F_{+}, F_{-}\right]\right) X \wedge \mathbf{1}
\end{aligned}
$$

In order to determine the stabilizer of $R$ in $\mathfrak{s o} V$ it seems prudent to study the Ricci curvature:

$$
\operatorname{Ric}(y \oplus Y, z \oplus Z):=\operatorname{tr}_{V}\left(x \oplus X \longmapsto R_{x \oplus X, y \oplus Y}(z \oplus Z)\right)
$$

Calculating the traces of the following expressions appearing in $R_{x \oplus X, y \oplus Y}(z \oplus Z)$ over $x \oplus X$

$$
\begin{aligned}
\left(F_{+} X \wedge F_{+} Y\right)(z \oplus Z) & =0 \oplus\left(g\left(F_{+} Z, X\right) F_{+} Y-g\left(F_{+} Y, Z\right) F_{+} X\right) \\
\left(x\left(F_{+}^{2}+\left[F_{+}, F_{-}\right]\right) Y \wedge \mathbf{1}\right)(z \oplus Z) & =\left(g\left(\left(F_{+}^{2}+\left[F_{+}, F_{-}\right]\right) Y, Z\right) x\right) \oplus\left(\begin{array}{c}
\ldots \\
\cdots
\end{array}\right) \\
\left(y\left(F_{+}^{2}+\left[F_{+}, F_{-}\right]\right) X \wedge \mathbf{1}\right)(z \oplus Z) & =\left(\begin{array}{c}
\ldots
\end{array}\right) \oplus\left(-y z\left(F_{+}^{2}+\left[F_{+}, F_{-}\right]\right) X\right)
\end{aligned}
$$

we obtain $g\left(F_{+}^{2} Y-\left(\operatorname{tr} F_{+}\right) F_{+} Y, Z\right)$ and $g\left(F_{+}^{2} Y+\left[F_{+}, F_{-}\right] Y, Z\right)$ as well as $-y z\left(\operatorname{tr} F_{+}^{2}\right)$ so that the Ricci endomorphism $g(\operatorname{Ric}(y \oplus Y), z \oplus Z):=\operatorname{Ric}(y \oplus Y, z \oplus Z)$ reads:

$$
\operatorname{Ric}(y \oplus Y)=-\left(\operatorname{tr}\left(F_{+}^{2}\right) y \oplus\left(\left[F_{+}, F_{-}\right]+\left(\operatorname{tr} F_{+}\right) F_{+}\right) Y\right)
$$

In this way we obtain the following upper and lower bound on the stabilizer of $R$ in $\mathfrak{s o} V$

$$
\mathfrak{s t a b} \operatorname{Ric} \supset \mathfrak{s t a b} R \supset \mathfrak{s t a b} F_{+} \cap \mathfrak{s t a b}\left[F_{+}, F_{-}\right] \cap \mathfrak{s o} V_{\circ}
$$

because the explicit formula for $R$ tells us that every $\mathfrak{X} \in \mathfrak{s o} V_{\circ}$ stabilizing $F_{+}$and $\left[F_{+}, F_{-}\right]$ stabilizes $R$. In order to proceed we need to be somewhat more specific about the special form of the endomorphism $F$ in the family of examples found by Meusers:

\section{Definition 6.8 (Meusers' Family of Examples)}

An endomorphism $F: V_{\circ} \longrightarrow V_{\circ}$ on a euclidian vector space $V_{\circ}$ of dimension $m-1 \geq 3$ is called special provided its diagonalizable symmetric part $F_{+}$has only two different eigenvalues of multiplicities 1 and $m-2$ respectively and every eigenvector $e \neq 0$ in the 1-dimensional eigenspace is cyclic for the skew symmetric part $F_{-}$of $F$ in the sense:

$$
V_{\circ}=\operatorname{span}\left\{e, F_{-} e, F_{-}^{2} e, F_{-}^{3} e, F_{-}^{4} e, \ldots\right\}
$$

Using the cyclicity of the eigenvector $e \neq 0$ of the symmetric part $F_{+}$of a special endomorphism $F$ under its skew symmetric part $F_{-}$we may construct a complete flag on $V_{\circ}$ via:

$$
\{0\} \subsetneq \operatorname{span}\{e\} \subsetneq \operatorname{span}\left\{e, F_{-} e\right\} \subsetneq \ldots \subsetneq \operatorname{span}\left\{e, F_{-} e, \ldots, F_{-}^{m-3} e\right\} \subsetneq V_{\circ}
$$


Up to the choice of signs there exists a unique orthonormal basis $e_{2}, \ldots, e_{m}$ of $V_{\circ}$ adapted to this flag, in this basis the matrix of the special endomorphism $F$ is tridiagonal of the form

$$
F \widehat{=}\left(\begin{array}{ccccc}
f_{1} & -f_{3} & & & \\
+f_{3} & f_{2} & -f_{4} & & \\
& +f_{4} & \ddots & \ddots & \\
& & \ddots & f_{2} & -f_{m} \\
& & & +f_{m} & f_{2}
\end{array}\right)
$$

where the parameters $f_{1}, \ldots, f_{m} \in \mathbb{R}$ are arbitrary except for $f_{1} \neq f_{2}$ and $f_{3}, \ldots, f_{m} \neq 0$ to ensure the cyclicity of the basis vector $e_{2}$. The special endomorphisms form in this way an $m$-parameter family of orbits in End $V_{\circ}$ under the action of the orthogonal group $\mathbf{O}\left(V_{\circ}\right)$. Extending this orthonormal basis to the orthonormal basis $\mathbf{1}, e_{2}, \ldots, e_{m}$ of $V$ we obtain the following explicit matrix for the Ricci endomorphism calculated in equation (12):

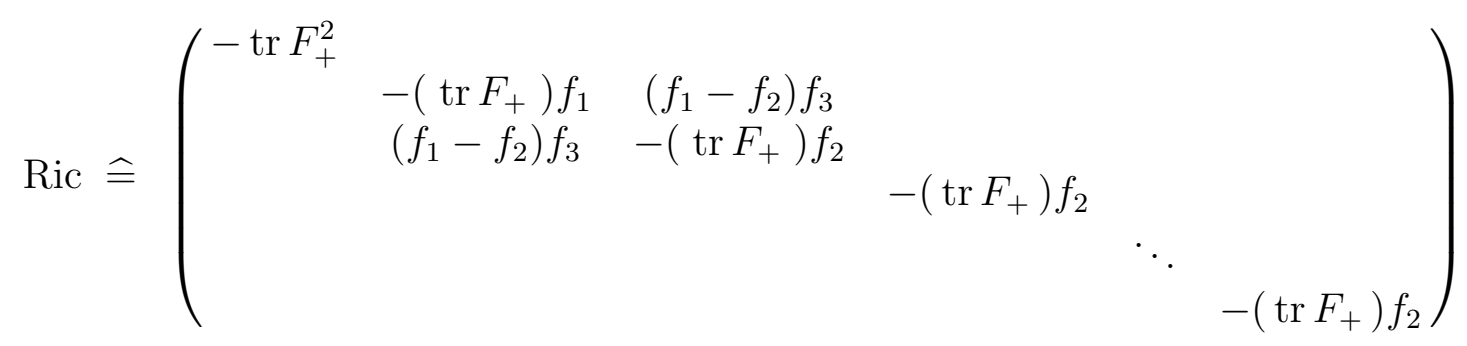

This matrix certainly has 4 different eigenvalues of multiplicities $m-3$ and 1, 1, 1 for a generic special endomorphism $F$, say for $f_{1}=1$ and $f_{2}=0$ these eigenvalues are 0 and $-1,-\frac{1}{2} \pm \frac{1}{2} \sqrt{4 f_{3}^{2}+1}$ ) respectively. For such a generic special endomorphism $F$

$$
\mathfrak{s t a b} \text { Ric }=\mathfrak{s t a b} F_{+} \cap \mathfrak{s t a b}\left[F_{+}, F_{-}\right] \cap \mathfrak{s o} V_{\circ}=\mathfrak{s o}\left\{1, e_{2}, e_{3}\right\}^{\perp}
$$

and equation (13) tells us that this agrees with the stabilizer of $R$ :

$$
\mathfrak{h}_{0}:=\mathfrak{s t a b} R=\mathfrak{s o}\left\{e_{4}, e_{5}, \ldots, e_{m}\right\}
$$

Because every $\mathfrak{X} \in \mathfrak{h}_{0}$ commutes with $F_{+}$, the comodule directional derivatives are given by

$$
\frac{\partial \mathfrak{X}}{\partial \mathbf{1}}=\left[\mathfrak{X}, F_{-}\right] \quad \frac{\partial \mathfrak{X}}{\partial X}=\left[\mathfrak{X}, F_{+} X \wedge 1\right]-F_{+}(\mathfrak{X} X) \wedge 1=0
$$

for all $X \in V_{\circ}$, in particular for $\mathfrak{X}:=e_{\mu} \wedge e_{\nu} \in \mathfrak{h}_{0}$ with $4 \leq \mu<\nu \leq m$ we obtain this way

$$
\frac{\partial}{\partial \mathbf{1}}\left(e_{\mu} \wedge e_{\nu}\right)=-\left(F_{-} e_{\mu} \wedge e_{\nu}+e_{\mu} \wedge F_{-} e_{\nu}\right) \equiv f_{\mu} e_{\mu-1} \wedge e_{\nu}
$$

modulo $\mathfrak{s o}\left\{e_{\mu}, \ldots, e_{m}\right\}$. Consequently the subalgebras in the stabilizer filtration (7) read

$$
\mathfrak{h}_{r}=\mathfrak{s o}\left\{e_{r+4}, \ldots, e_{m}\right\}
$$

for all $r=0, \ldots, m-4$ due to our cyclicity assumption $f_{3}, \ldots, f_{m} \neq 0$. In consequence the affine homogeneous spaces of dimension $m$ associated to generic special endomorphisms 
$F: V_{\circ} \longrightarrow V_{\circ}$ on euclidian vector spaces $V_{\circ}$ of dimension $m-1$ have Singer invariant $m-4$, because $\mathfrak{h}_{m-5} \neq\{0\}$, but $\mathfrak{h}_{m-4}=\mathfrak{h}_{\infty}=\{0\}$.

Before closing this section we want to calculate the Spencer cohomologies in this family of examples of affine spaces with large Singer invariant. With all directional derivatives $\frac{\partial \mathfrak{X}}{\partial X}=0$ in directions $X \in V_{\circ}$ vanishing we may use the isomorphism (10) to reduce this calculation to the calculation of the Spencer cohomology of the comodule $\mathfrak{h}^{\bullet}$ considered as a comodule over Sym $V / V_{\circ}=\operatorname{Sym} \mathbb{R}$. By definition the comodule $\mathfrak{h}^{\bullet}$ is the direct sum of the successive quotients $\mathfrak{h}_{r-1} / \mathfrak{h}_{r}$, which are spanned for $r>0$ by the bivectors $e_{r+3} \wedge e_{\nu}+\mathfrak{h}_{r}$ with $r+3<\nu \leq m$. The only non-trivial directional derivatives are injective for $r>0$

$$
\frac{\partial}{\partial \mathbf{1}}: \quad \mathfrak{h}_{r} / \mathfrak{h}_{r+1} \longrightarrow \mathfrak{h}_{r-1} / \mathfrak{h}_{r}, \quad e_{r+4} \wedge e_{\nu}+\mathfrak{h}_{r+1} \longmapsto f_{r+3} e_{r+3} \wedge e_{\nu}+\mathfrak{h}_{r}
$$

with cokernel spanned by $e_{r+3} \wedge e_{r+4}+\mathfrak{h}_{r}$. In summary we have proved the following lemma:

\section{Lemma 6.9 (Singer Invariant and Spencer Cohomology)}

Consider a special endomorphism $F: V_{\circ} \longrightarrow V_{\circ}$ on a euclidian vector space $V_{\circ}$ of dimension $m-1 \geq 3$ in the sense of Definition 6.8 and let $e_{2}, \ldots, e_{m}$ be the essentially unique orthonormal basis of $V_{0}$, in which $F$ takes the trilinear form (14). The euclidian Lie algebra $\mathfrak{g}=\mathbb{R} \oplus V_{\circ}=V$ associated to the special endomorphism $F$ has Singer invariant

$$
\operatorname{Singer}(\mathfrak{g})=m-4
$$

provided the Ricci endomorphism of $\mathfrak{g}$ has 4 different eigenvalues. For such a generic special endomorphism $F$ the Spencer cohomology $H^{r, o}(\mathfrak{h})$ of the associated comodule $\mathfrak{h} \bullet$ over the coalgebra Sym $V^{*}$ is a free $\Lambda^{\circ} V_{\circ}^{*}$-module of rank 1 for all $r=1, \ldots, m-4$ with isomorphism

$$
\Lambda^{\circ-1} V_{\circ}^{*} \stackrel{\cong}{\longrightarrow} H^{r, \circ}(\mathfrak{h}), \quad \eta \longmapsto\left[\mathbf{1}^{\sharp} \wedge \eta \otimes\left(e_{r+3} \wedge e_{r+4}+\mathfrak{h}_{r}\right)\right]
$$

where $\mathbf{1}^{\sharp} \in V^{*}$ is the linear form $\mathbf{1}^{\sharp}(x \oplus X):=x$. In particular the interesting Spencer cohomologies $H^{r, 1}(\mathfrak{h})$ for $r=1, \ldots, m-4$ are all one-dimensional.

The remarkable conclusion of our calculation of the Spencer cohomology of Meusers' examples of Riemannian homogeneous spaces with large Singer invariant is that this specific family of examples is in a sense maximal in the moduli space $\mathfrak{M}(\mathfrak{s o} V)_{\infty}$ : Every vector formally tangent to the moduli space $\mathfrak{M}(\mathfrak{s o} V)_{\infty}$ in a point corresponding to a Riemannian homogeneous space in Meusers' family is integrable to a real deformation by changing one of the parameters $f_{5}, \ldots, f_{m}-1 \neq 0$ of this family.

\section{References}

[AS] Ambrose, W. \& Singer, I. M.: On Homogeneous Riemannian Manifolds, Duke Mathematical Journal 25 (1958), 647-669. 
[BCG] Bryant, Chern, Gardner, Goldhhmidt, Griffiths: Exterior Differential Systems, MSRI Lecture Notes 18, Springer (1990).

[H] Helgason, S.: Differential Geometry, Lie Groups and Symmetric Spaces, Graduate Studies in Mathematics, American Mathematical Society (2001).

[KT] Kiyota, Y. \& Tsukada, K.: Curvature Tensors and Singer Invariants of FourDimensional Homogeneous Spaces, Commentationes Mathematicae Universitatis Carolinae 40 IV (1999), 723-733.

[K] Kobayashi, S.: Transformation Groups in Differential Geometry, Classics in Mathematics 70, Springer (1995).

[KN] Kobayashi, S. \& Nomizu, K. : Foundations of Differential Geometry I, Interscience Publishers (1963).

[K1] Kowalski, O.: Counter-Example to the "Second Singer's Theorem", Annals of Global Analysis and Geometry 8 II (1990), 211-214.

[K2] Kowalski, O.: On Strictly Locally Homogeneous Riemannian Manifolds, Differential Geometry and its Applications 7 II (1997), 131-137.

[O] Opozda, B.: A Classification of Locally Homogeneous Connections on 2-Dimensional Manifolds, Differential Geometry and its Applications 21 II (2004), 173-198.

[M] Meusers, C.: High Singer Invariant and Equality of Curvature, Bulletin of the Belgian Mathematical Society - Simon Stevin 9 / IV (2002), 491—502.

[NT] Nicolodi, L. \& Tricerri, F.: On Two Theorems of I. M. Singer about Homogeneous Spaces, Annals of Global Analysis and Geometry 8 II (1990), 193-209.

[S] Singer, I. M.: Infinitesimally Homogeneous Spaces, Communications in Pure and Applied Mathematics 13 (1960), 685-697

[Tr] Tricerri, F.: Locally Homogeneous Riemannian Manifolds, Rendiconti del Seminario Matematico Università e Politecnico di Torino 50 IV (1992).

[Ts] Tsukada, K.: The Moduli Space of Locally Homogeneous Spaces and Locally Homogeneous Spaces which are not Locally Isometric to Globally Homogeneous Spaces, Proceedings of the Satellite Conference of ICM in Berlin, Brno 1998, Masaryk University (1999), 149-159.

[W] Weingart, G.: An Introduction to Exterior Differential Systems, Geometry of Manifolds with Non-negative Sectional Curvature, Lecture Notes in Mathematics 2110 (2014), 117-196 